LAWRENCE LIVERMORE N A T IO N A L LABORATORY
A Unique U.S. Approach for Accelerator-Driven Warm Dense Matter Research--Preliminary Report

B. G. Logan, R. C. Davidson, J. J. Barnard, R. Lee

December 27, 2004 
This document was prepared as an account of work sponsored by an agency of the United States Government. Neither the United States Government nor the University of California nor any of their employees, makes any warranty, express or implied, or assumes any legal liability or responsibility for the accuracy, completeness, or usefulness of any information, apparatus, product, or process disclosed, or represents that its use would not infringe privately owned rights. Reference herein to any specific commercial product, process, or service by trade name, trademark, manufacturer, or otherwise, does not necessarily constitute or imply its endorsement, recommendation, or favoring by the United States Government or the University of California. The views and opinions of authors expressed herein do not necessarily state or reflect those of the United States Government or the University of California, and shall not be used for advertising or product endorsement purposes.

This work was performed under the auspices of the U.S. Department of Energy by University of California, Lawrence Livermore National Laboratory under Contract W-7405-Eng-48. 


\title{
A Unique U.S. Approach for Accelerator-Driven Warm Dense Matter Research--Preliminary Report*
}

\author{
Grant Logan', Ron Davidson ${ }^{3}$, John Barnard², Richard Lee ${ }^{2}$ \\ 1. LBNL 2. LLNL 3. PPPL \\ Preliminary version September 28, 2004 \\ This first preliminary white paper will be updated periodically as we learn more from further \\ analysis, and to take into account input we get from various workshops, such as the one on \\ HEDP planned at LBNL on Oct. 26-29, 2004.
}

\section{Introduction}

The warm density matter regime of high energy density physics $[1,2,3]$ has a high scientific discovery potential for the properties of plasmas at high densities and pressures and at moderate temperatures $(k T)$ in which the Coulomb interaction energy between plasma particles exceed $k T$. This leads to correlations in the plasma characterized by the dimensionless "coupling" parameter $\square>1$, where $\square$ is defined by

$$
\square=\frac{q^{2} n^{1 / 3}}{k T}
$$

Here $q$ is the effective ion charge and $n$ the ion density. Strongly-coupled plasmas with $\square>1$ are difficult to study analytically and by numerical simulation. Many astrophysical systems (e.g., brown dwarfs, and giant planets) and inertial fusion plasmas in the beginning stages of compression fall into this regime. There is an opportunity to develop improved understanding and models through accurate measurements of properties in the large parameter space of temperature and density where data is currently limited or non-existent. X-ray free-electron lasers (Fourth generation light sources), ultra-short pulse and high energy optical lasers, pulsedpower z-pinch X-ray sources, and high explosives are all capable of producing warm dense matter conditions at various temperatures, pressures, and sample sizes. Therefore, the challenge is not how to create warm dense matter conditions, but to create it so that it's fundamental properties can be best studied. The goal is to advance this field of science through a variety of complementary facilities and methods which offer several combinations of desirable attributes:

- Precise control and uniformity of energy deposition;

- Large sample sizes compared to diagnostic resolution volumes;

- A benign environment for diagnostics (low debris and radiation background);

- High shot rates (10/hour to $1 /$ second) and multiple beamlines/target chambers;

- Sites with easy access for broad participation by university scientists and students; and with the technical support for designing and fielding targets for qualified experiments.

\footnotetext{
* Work performed under the auspices of the U.S. Department of Energy under University of California contract W-7405-ENG-48 at LLNL, University of California contract DE-AC03-76SF00098 at LBNL, and contract DEFG0295ER40919 at PPPL.
} 
Dedicated, modest energy facilities are needed for developing new experimental techniques and diagnostics as well as the largest facilities for the most demanding target requirements. The recent report [4] of the National Task Force on High Energy Density Physics notes: “.... range of facilities is essential to perform experiments at increasing energy/current in order to develop experimental and diagnostic techniques before carrying out experiments on the larger facilities such as the National Ignition Facility (NIF) or the Z/ZR facility, where operating costs are high." Because of the high costs and limited number of shots available to external users on the large facilities, modest scale facilities, as the one described in this white paper, will be crucial "to validate simulation capabilities, to gather physical insight, and to develop target fabrication expertise, that will ensure success of the limited number of experiments possible on the largest facilities."

A recent white paper [5] for DOE Science Director Ray Orbach describes the general scientific motivation for high energy density physics, and how LBNL could contribute to selected key scientific questions in a variety of ways, including the use of heavy-ion beam accelerators. That white paper described the physics motivation for considering the use of heavy ions to heat warm dense matter:

"Intense ion beams, if compressed to short enough pulses and delivered to small enough focal spots, could provide an important new tool for exploring high energy density plasmas (HEDP) in a different, complementary way to using lasers. This is because of the unique energy deposition property of ion beams, namely, that in very dense plasmas compared to the beam ion density, ions are observed to slow down in straight-line trajectories due to classical processes. At high energies, the ion range can be large compared to the optical mean-free-paths in solid-density plasmas, allowing ion energy deposition at the Bragg peak to be well-inside targets that would be opaque to laser light or soft-x-ray radiation. In contrast, lasers deposit their energy at plasma critical densities $<<$ solid densities, where $\square_{\text {laser }} \backslash \square_{\mathrm{p}}$. Here, $\square_{\mathrm{p}}$ is the plasma frequency. The deep penetration of high-energy ion beam heating allows more flexibility in some types of targets that can be used for dense plasma science."

Recent research in the heavy-ion fusion program describes how the use of heavy-ion beams with energies just above the Bragg peak in $\mathrm{dE} / \mathrm{dx}$ (ion energy loss per unit range) can maximize heavy ion deposition power density and uniformity simultaneously [6]. As a guiding principle we believe this method will provide a unique and affordable approach for a U.S. heavyion driven HEDP user facility.

The purpose of the present white paper is to address more specifically the opportunities and plans to develop a US accelerator-driven HEDP user facility, namely:

(1) How modest-cost U.S heavy-ion accelerators and experimental target areas can best be designed to meet the needs of HEDP users (Section II), and compare with the HEDP-use potential of other accelerators particularly, GSI-SIS18/100.

(2) Describe how certain types of targets and diagnostics can be well matched to heavyion drivers to explore warm dense matter where data is most needed to test stronglycoupled plasma models and determine equations of state (Section III) 
The above-mentioned favorable deposition properties of heavy ions, and a German government decision to upgrade a heavy-ion linac and storage ring at GSI in part for warm dense matter studies, provides motivation for an intended collaboration called High Energy Density Matter Generated by Heavy Ion Beams (HEDgeHOB) [7], including U.S. participation under the auspices of a DOE-German government agreement for collaboration in dense plasma science [8]. During the next 8 to 10 years that it will take before the GSI upgrade is productive, the GSI group will be testing diagnostics using less intense heavy ion pulses from their present machine, and there is opportunity for the U.S heavy-ion driven HED program to benefit from this collaboration. In particular, the Germans will be learning how to use transmission, energy loss, and charge state measurements of the heavy-ion beam itself as a probe for warm dense matter, and there will also be the application of an intense short-pulse laser to image dense heavy-ion beam targets [7]. However, international collaboration will not benefit the U.S. science program without U.S. state-of-the-art ion beam facilities to contribute to the underlying science through a strong domestic research program in high energy density physics.

This white paper is laid out as follows: Section II describes how U.S. accelerators with low cost, moderate energy ions can provide a unique approach to heating warm dense matter that would complement the GSI capability and greatly enrich the mutual collaboration. Section III describes opportunities and plans for targets, chambers and diagnostics which will provide an excellent match for US accelerator capabilities to advance HEDP science. Conclusions are given in Section IV.

\section{U.S. approach proposed with moderate energy ion accelerators}

\section{Maximizing desirable attributes for advancing warm dense matter science}

The principal goal is a dedicated, modest energy facility, such as recommended by the National HEDP Task Force, which could advance the warm dense matter regime of HEDP science in the U.S. by allowing many users to explore new experimental techniques at low cost and with many shots, which could later lead to qualified experiments on high energy NNSAfunded facilities. As discussed in the introduction, there are several candidate drivers for such facilities, so the strategy is to develop an approach which can maximize the five desirable attributes for scientific productivity described in the introduction, and at minimum cost. Appendix 1 contains excerpts from the National HEDP Task Force Report [4] on the top-level scientific question regarding the prerequisite heavy ion beam compression and focusing required for the U.S. accelerator-driven HEDP approach described in this white paper, and also describes a ten-year research plan with intermediate milestones to address the principal beam physics questions. The intense beam-plasma regimes in the accelerator and in the longitudinal drift compression region are themselves interesting "extreme states of matter" to study as well as the warm dense matter target physics. Building upon the National Task Force report described in Appendix 1, this white paper provides additional description of the plans, issues and opportunities for the experimental targets, diagnostics and facility operation so as to optimize the scientific productivity and utility of this approach for warm dense matter research. In parallel with the accelerator/beam compression experiments, we plan to explore candidate experimental target and chamber designs that can best utilize the ion beams as they progress up in intensity at each stage. We also plan to gradually develop an appropriate suite of diagnostics, starting with 
existing ion and laser beams in other facilities, so as to be able to field initial HEDP user experiments beginning in about 5 years.

\section{Maximizing precision control of energy deposition}

To maximize precision control of the energy deposition profile, it is proposed to use ion beams with predictable deposition physics [5]. The energy loss and charge state of the transmitted ions can also be used as a diagnostic for warm density matter energy density and temperature. Multi-stage accelerators with agile voltage waveform control will be used to precisely tailor the longitudinal energy distribution of the ion beams, and therefore manipulate the energy deposition and pressure profiles in the targets. Fast ions (fast compared to average target electron velocities) deposit energy by slowing down on target electrons at a rate $\mathrm{dE} / \mathrm{dx}$ that increases as the ions slow down until the ion speed drops below the average electron velocity (bound electron velocities for cold target materials, or electron thermal velocity in fully ionized targets). At such a point, sometimes referred to the Bragg peak, $\mathrm{dE} / \mathrm{dx}$ reaches a maximum and then declines to zero as the ion stops. Figure 1 plots a typical curve of $\mathrm{dE} / \mathrm{dx}$ as a function of penetration depth in a target for a fast heavy ion with an initial kinetic energy starting well above the peak in $\mathrm{dE} / \mathrm{dx}$, such as the case in multi-GeV heavy ions in RF linac/storage ring accelerators for nuclear physics studies at GSI [7]. As a complementary approach to the GSI program, it is proposed to use ion beams entering the targets at moderate kinetic energies just above the energy where the ion deposition rate $\mathrm{dE} / \mathrm{dx}$ is maximum for a given ion species and target condition, to maximize both the rate and uniformity of energy deposition [6]. The moderate ion energies required for heating at the peak in $\mathrm{dE} / \mathrm{dx}$, in the range of a few $\mathrm{MeV}$, allows this U.S. approach to use moderate accelerator voltages, lengths, and cost.

Table 1 lists ion ranges in solid cold titanium foils for selected light ions at various initial energies, and the corresponding ion energies at the $\mathrm{dE} / \mathrm{dx}$ peak. The proposed use of ions just above the peak in $\mathrm{dE} / \mathrm{dx}$ minimizes the accelerator length and cost, but requires more ions to deliver a given energy to the target compared to using ions at much higher energies. The current density of ions at the moderate energies required to heat targets to several $\mathrm{eV}$ temperature, when also compressed to sufficiently short pulses that the target conditions remain uniform for measurements, leads to the necessity to compress and focus the ion bunches within a background plasma in order to neutralize the beam space charge between the accelerator and the target $[4,9$, 10]. 


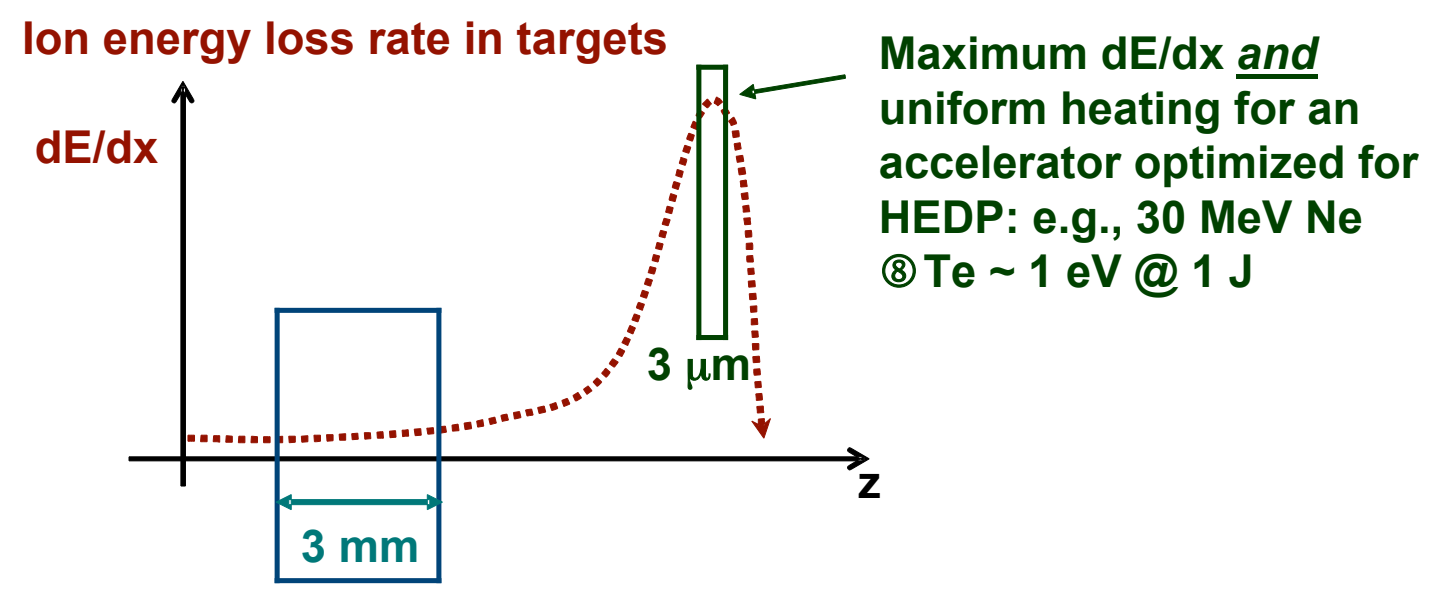

\author{
GSI: e.g., $30 \mathrm{GeV}$ U lons \\ (8) massive targets, \\ (8)Te 1 eV@1 kJ
}

Figure 1 A typical curve of ion energy loss rate versus depth $\mathrm{z}$ into a target. It is proposed to inject ions at moderate kinetic energies just above the energy of peak $\mathrm{dE} / \mathrm{dx}$, such that the peak in $\mathrm{dE} / \mathrm{dx}$ occurs in the center of the target for maximum uniformity. The targets should still be less than the total ion range, to allow the energy loss of transmitted ions to be used as a diagnostic for energy deposition, and the charge state of the transmitted ions for target temperature.

\begin{tabular}{|c|c|c|c|}
\hline Beam ion & $\begin{array}{c}\text { Initial energy } \\
\text { (MeV) }\end{array}$ & $\begin{array}{c}\text { Range in solid titanium } \\
\text { (microns) }\end{array}$ & $\begin{array}{c}\text { Energy at dE/dx peak } \\
\text { (MeV) }\end{array}$ \\
\hline $\mathrm{H}$ & 1 & 11.4 & 0.11 \\
\hline $\mathrm{He}$ & 1 & 3.1 & 0.7 \\
\hline $\mathrm{N}$ & 10 & 5.8 & 7.7 \\
\hline $\mathrm{Ne}$ & 16 & 6.3 & 15 \\
\hline
\end{tabular}

Table 1 Nominal ion ranges and energies at $\mathrm{dE} / \mathrm{dx}$ peaks in cold titanium target foils for a few selected light ions for a moderate energy and cost accelerator for HEDP studies (from Ref. 6). Exact ranges and energies at the $\mathrm{dE} / \mathrm{dx}$ peak depend on the temperature and degree of ionization of the target.

\title{
Large sample sizes compared to diagnostic resolution scales
}

Typically, ion focal spot radii of approximately $1 \mathrm{~mm}$ are expected, which is large enough in the radial direction that the beam heating can be uniform radially within a few hundred microns of the axis where local target properties can be measured. For solid target foils with thicknesses less than the few-micron ion ranges shown in Table 1, hundred-picosecond-scale ion pulses are desired to limit the effects of hydrodynamic expansion during the measurements. A few years will be required before the beam compression research described in Appendix 1 would 
evaluate the potential to achieve sub-nanosecond pulses, and it is planned to drive targets of 10's to 100's of micron thicknesses that could work with longer, nominally $1 \mathrm{~ns}$, ion pulses. In addition, such minimum thicknesses will make it easier for diagnostics to resolve central portions of the target sample. However, thicker targets at solid density imply longer ion ranges, higher ion kinetic energy and mass at peak $\mathrm{dE} / \mathrm{dx}$, and thus greater accelerator length and cost. Therefore, it is planned to start warm dense matter experiments with low average density foam or wire-array targets which can be 10 to 100 times thicker for the same ion range and pulse energy, and with corresponding longer hydro-expansion times, assuming the ion heating homogenizes the resulting plasma. Similarly, it may be possible to make dense gas jets at low temperatures where small clusters or droplets form within the jets (two-phase flows), resulting in uniform sub-solid density plasmas after isochoric beam heating for equation of state studies. Fig. III.x in Section III illustrates how plasma densities lower than solid, densities down to $0.01 \mathrm{x}$ solid density or lower, can still provide discriminating data to test equation-of-state models, and so use of low density foams or gas jets can keep the initial accelerator pulse energy requirements and costs low. A key design issue is how fast and how uniformly the interstitial voids in such initially heterogeneous targets fill in with ion beam heating. Hydrocodes with Advanced Mesh Refinement are available to evaluate this issue. Use of low-density foam targets will allow us to begin warm density matter research with pulses initially as low as $1 \mathrm{~J}$ and then upgrade the accelerator pulse energy in steps up to about $100 \mathrm{~J}$. This is a prudent way to manage the investment cost and technical risk while developing experimental techniques at each stage.

\section{Benign environment for diagnostics and personnel safety}

Moderate energy ion pulses at peak $\mathrm{dE} / \mathrm{dx}$ as indicated in Table 1 should not generate significant hazards or facility activation from neutrons or gammas produced by the ion beams themselves. (In contrast, at multi-GeV energies, heavy ions create neutrons and radioactive nuclear fragments, which can lead to significant facility shielding expenses). In experiments needing a short-pulse laser for X-ray diagnostics, multi-MeV electrons can be produced which can require some gamma shielding, but not likely neutron shielding to prevent activation. Also, with the modest pulse energies envisioned here, only about ten micrograms of target material are vaporized each shot, making target debris from a large number of shots and rapid bursts of shots tolerable before diagnostic windows likely need to be cleaned or replaced.

\section{High shot rates/multiple experimental chambers/dedicated site}

As in conventional accelerators serving multiple experimental areas for multiple users, we envision an ion beam deflector using dipole magnets, or equivalent rapid switching, to support three or more experimental target chambers with one accelerator. As long as pulsed energy charging supply currents are designed for high shot rates, accelerators of the type envisioned here have been demonstrated to sustain $1 \mathrm{~Hz}$ pulse rates, and can likely be designed for $10 \mathrm{~Hz}$ with the low time-average duty factors for the required beam loading. Thus accelerator constraints will likely support pulse rates as high as experimental data acquisition rates require, even for more than one chamber. For solid targets mounted on in-vacuum target wheels containing 100 or more targets, 100-pulse trains at 10 shots per minute rates might productively be used, and pulse rates of $1 \mathrm{~Hz}$ might be useful with gas jet targets, as long as diagnostic data acquisition rates are designed to keep up with such rates. If such high shot rates 
were available, advantage would be taken of statistical averaging to reduce the measurement error bars wherever possible. The data rates required to optimize scientific productivity will not be known until experiments are carried out . Since it doesn't add much cost to design accelerators for high shot rates, the goal is to provide high-shot-rate capability in nominal 100shot bursts of 0.1 to $1 \mathrm{~Hz}$.

As pointed out in the National HEDP Task Force [4], and discussed further in Section III, university users need considerable on-site assistance with designing, fabricating, and fielding their experimental targets, and with diagnostic setup and data acquisition around the experimental chambers. This user support will work best if the funding for the facility and onsite support is dedicated to the warm dense matter science program as the primary mission. A non-weapons lab site can also facilitate access for university users and students.

Figure 2 shows a conceptual example of an accelerator-driven HEDP user facility, with a single ion beam linac supplying several target chambers through a beam director (three chambers are shown in this example). The beam intensities after acceleration are high enough that beam space charge must be neutralized in low density $\left(\sim 10^{12} \mathrm{~cm}^{-3}\right)$ pre-formed plasma from the exit of the accelerator up to each target. Each type of experimental target is expected to require an integrated design for the target holder, plasma source, diagnostics, final focus, and plasma filled drift compression line, as illustrated in Fig 2. The beam director may contain one or more variable dipole magnets to provide beam deflection on demand into any chamber, and to provide first-order chromatic correction for optimal beam focus. In addition, the accelerator is short enough $(<3$ meters) to allow the option to have the linac pivot around the beam director, eliminating or reducing the bend angles required for each chamber. (Numerous experimental diagnostics would make moving the target chambers instead very time-consuming).

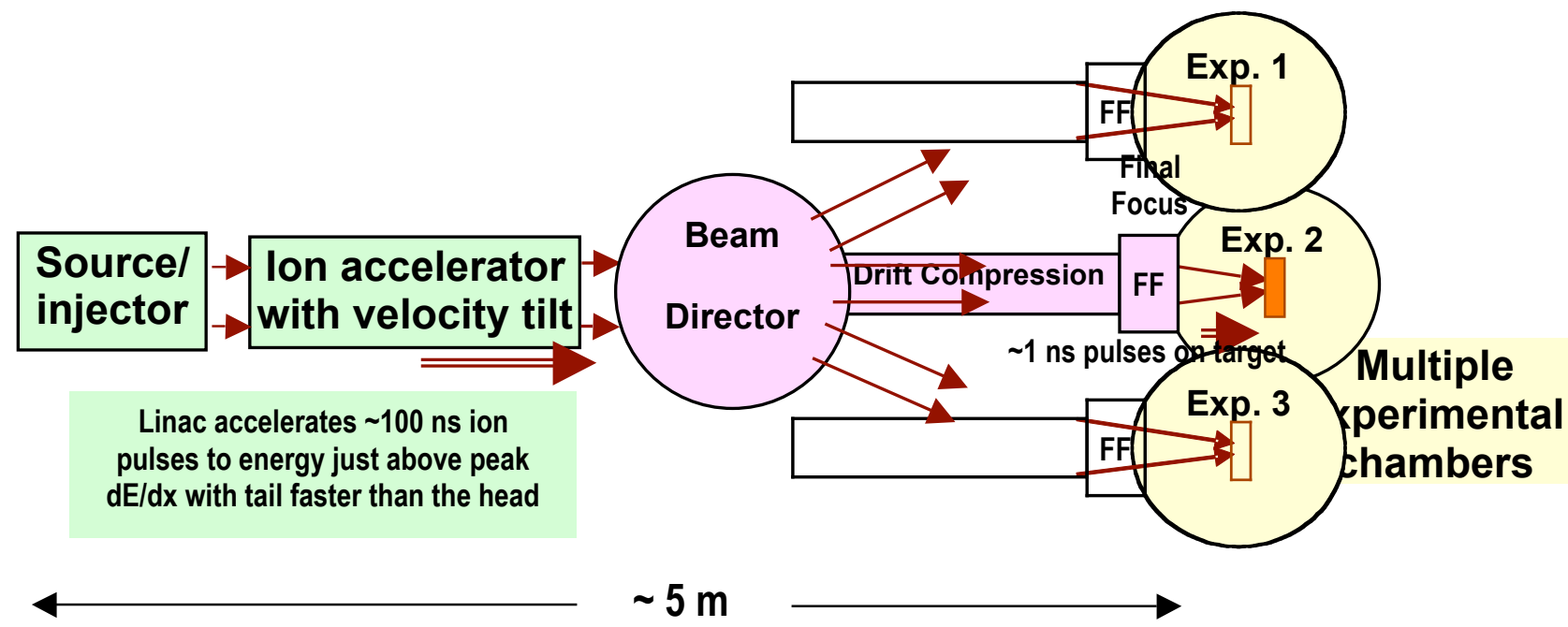

Fig. 2: Schematic of a modest size ion linac driving multiple HEDP experimental chambers with ions at a few $\mathrm{MeV}$ near the peak in $\mathrm{dE} / \mathrm{dx}$. Ion pulses are longitudinally drift compressed and focused within low density background plasma between the accelerator and the targets. 
The ion accelerator section depicted in Fig. 2 takes the beam from the injection energy up to two-thirds or more of the desired energy above the peak in $\mathrm{dE} / \mathrm{dx}$ for a chose ion species, with the remaining energy for the velocity tilt added by a special induction module. The induction module with agile waveform control provides a rising voltage pulse for the subsequent longitudinal beam compression in the plasma-filled drift lines to each target. For the first (uniform) part of the beam acceleration after injection, several types of accelerators are being considered, including multi-stage induction, helical pulse lines, and RF. There is also a single voltage gap version of induction, called the Induction Voltage Adders (IVA). All of these options will be assessed in a workshop for accelerator-driven HEDP in October 2004. All of these options will require neutralized beam pulse compression and final focusing to meet desired HEDP conditions as described above, and any option would go through incremental stages of experiments for neutralized pulse compression as described above and in Appendix 1. One example of a sequence of three accelerator and beam compression experiments based on induction, called Neutralized Drift Compression Experiments (NDCX series) is shown in Figure 3. In these sketches, only one target chamber is shown because they illustrate beam compression experiments. In the middle step labeled NDCX IIb and the last step NDCX-III, warm dense matter experiments would utilize several target chambers as illustrated in Fig. 2.
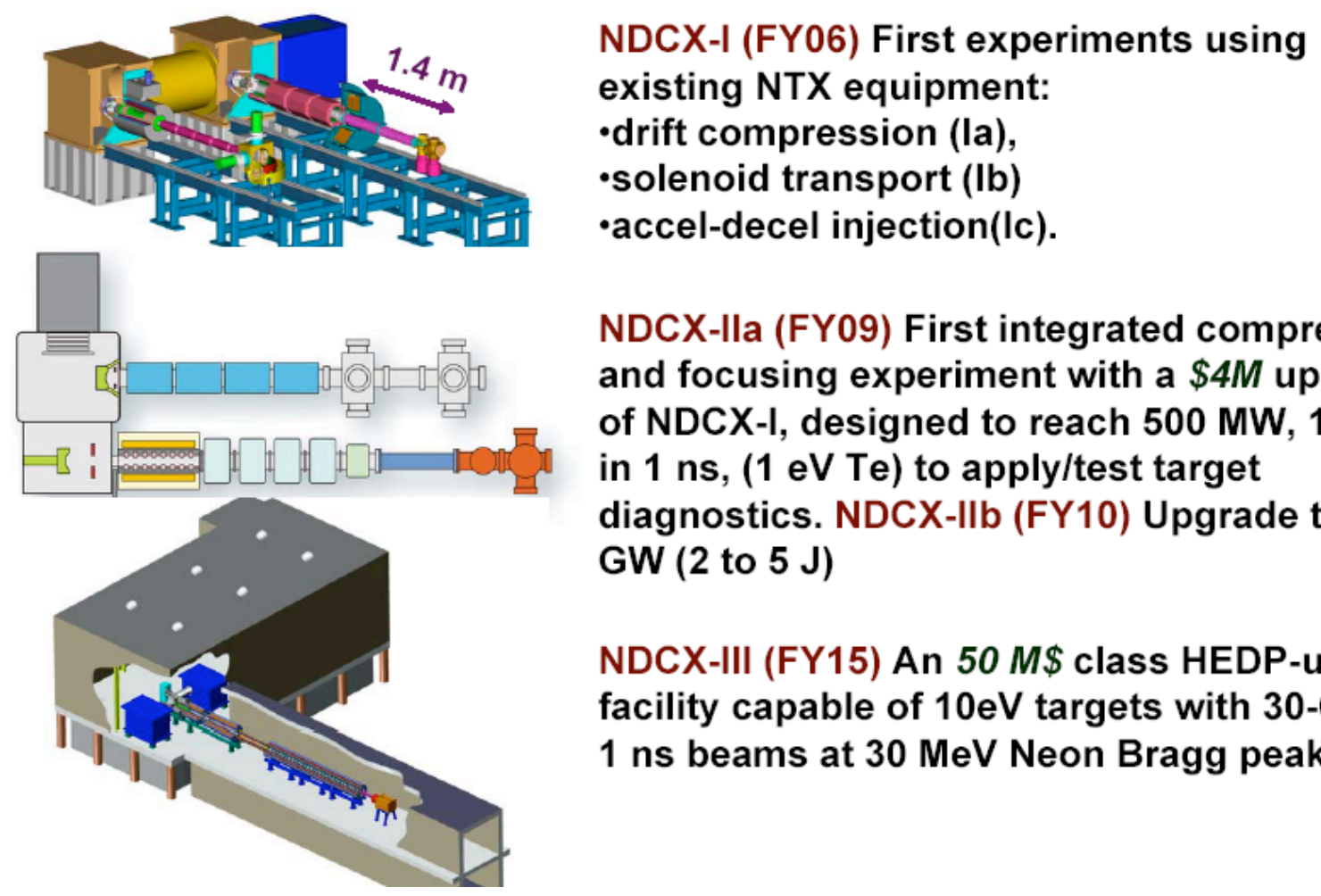

NDCX-Ila (FY09) First integrated compression and focusing experiment with a $\$ 4 M$ upgrade of NDCX-I, designed to reach $500 \mathrm{MW}, 1 \mathrm{MeV}$ in $1 \mathrm{~ns},(1 \mathrm{eV} \mathrm{Te})$ to apply/test target diagnostics. NDCX-IIb (FY10) Upgrade to 2-5 $\mathrm{GW}(2$ to $5 \mathrm{~J})$

\section{NDCX-III (FY15) An $50 M \$$ class HEDP-user facility capable of $10 \mathrm{eV}$ targets with $30-60 \mathrm{GW}$, $1 \mathrm{~ns}$ beams at $30 \mathrm{MeV}$ Neon Bragg peak.}

Figure 3: A sequence of neutralized drift compression experiments with increasing capability for warm dense matter experiments. The steps labeled NDCX-IIb and NDCX-III would be configured with multiple experimental chambers (only one chamber shown) for warm dense matter studies as in Fig. 2. 
The capability of NDCX drivers for warm dense matter studies approximately parallels the capability of GSI: the Germans are now working on upgrading the linac injection and vacuum system in the present SIS 18 ring with a goal to store $\sim 10 \mathrm{X}$ more ions, which may take several more years, if the recent rate of progress is maintained. If successful, the SIS18 should then deliver roughly a kilojoule of $300 \mathrm{MeV} / \mathrm{u}$ heavy ions compressed to $100 \mathrm{ns,} \mathrm{which} \mathrm{they}$ calculate should be sufficient to reach $1 \mathrm{eV}$ average target temperatures in isochoric heating experiments. The German government has approved construction of a larger upgrade, called SIS100 , which should be delivering $40 \mathrm{~kJ}$ beams of higher energy $0.5-1 \mathrm{GeV} / \mathrm{u}$ heavy ions to targets approximately 7-8 years after construction funding starts, hopefully beginning next year. The SIS-100 is estimated to be able to achieve $20 \mathrm{eV}$ in isochoric heating experiments [7]. The reason achievable target temperatures are expected to be similar between SIS-18 and NDCX-IIIIb, and between NDCX-II and SIS-100, despite the fact that the corresponding GSI beam pulse energies are almost $1000 \mathrm{X}$ higher, is because the GSI ion ranges are also $1000 \mathrm{X}$ longer than in the NDCX machine using lower energies where $\mathrm{dE} / \mathrm{dx}$ peaks (see Fig. 1). SIS is intended to be a multi-user facility, so that the warm density matter group at GSI has to share the beam time, and this is also why they have to accept the constraints of the higher beam energies optimized for nuclear physics [7].

\section{Contributions of NDC beam compression and focusing research to basic beam/plasma physics}

A very important aspect of the ion driven HEDP and the heavy ion fusion program is its strong synergism with other areas of physics research with high discovery potential. Prominent among these areas of connection are: advanced nonlinear dynamics (collective interaction processes, chaos); high energy and nuclear physics (accelerator physics); non-equilibrium statistical mechanics of charged particle systems; nonneutral plasma physics (space-charge effects, theoretical techniques); magnetic fusion plasma physics (beam-plasma interactions, diagnostic techniques); advanced computing (algorithms, massively parallel computations); and atomic physics (ionization and stripping cross sections). Very importantly, the detailed theoretical and experimental investigations of the intense charged particle beams carried out in the ion-driven HEDP program contribute significantly to the foundations of accelerator physics that find applications in other areas of research, including high energy and nuclear physics. Areas of common scientific interest include: halo particle production and control; the effects of intense space-charge fields; electron cloud effects; the use of background plasma to focus intense ion beams; and beam manipulation and compression, to mention a few examples. The high spacecharge fields associated with the intense ion beams encountered in ion driven HEDP and heavy ion fusion assure that the scientific challenges associated with collective excitations, electron cloud effects, beam-plasma interactions, etc., have high intellectual challenge and impact potential in other important areas of beam science research.

\section{Opportunities to optimize targets, chambers and diagnostics for warm dense matter studies using moderate energy ion accelerators.}

The interest in the warm dense matter regime arises because in dense plasmas the atoms and/or ions will start to behave in a manner that is intrinsically coupled to the plasma. That is, the plasma starts to exhibit long- and short-range order due to the correlating effects of the 
atoms/ions. This intriguing regime where the plasma can no longer be considered a thermal bath and the atoms are no longer well described by their isolated atom behavior provides a tremendous challenge to researchers. In the limit of dense cool plasmas one obviously arrives at the threshold of condensed matter. Here the problem has changed from a perturbative approach to ground-state methods where complete renormalization of the atom/ion and it environment is essential.

From the prospective of plasma studies the defining quantity is the coupling parameter $\square$, i.e., the ratio of the inter-atomic potential energy to the thermal energy given by the equation:

$$
\square=\frac{Z^{2} e^{2}}{r_{0} k T} \text { with } r_{0}=\frac{3 Z}{\square\left[n_{e}\right.} \text { 目 }^{1 / 3}
$$

where $Z$ is the ion charge and $r_{o}$ is the interparticle spacing given in terms of the electron density $n_{e}$.

The regions of interest span the density-temperature phase space going from modestly coupled $(\square \leq 1)$ to strongly coupled $(\square>1)$, while bridging the transition regimes between solid to liquid to plasma.
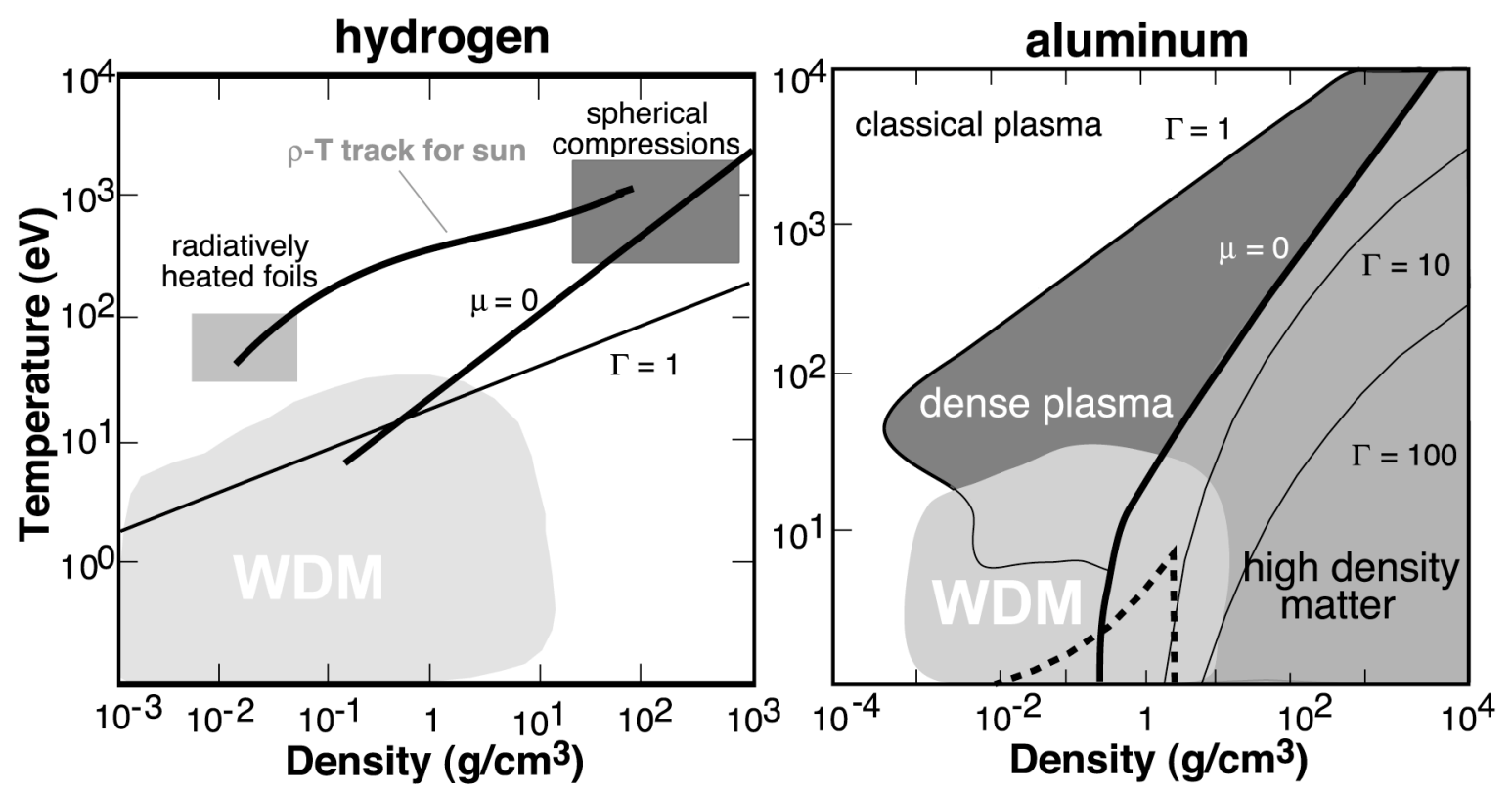

Figure 4. The temperature-density phase diagram for hydrogen on the left and aluminum on the right. The relevant regimes are noted, as are the various values of the coupling $\square$. The regions of greatest uncertainty are roughly noted by the light gray areas. Also indicated is the region where degeneracy will become important: it is the region to the right of the line where the chemical potential $\mu=0$. The hydrogen data is taken from a compilation of data from the NRL Plasma Formulary [22] while the aluminum data is derived from the QEOS formalism [23].

In figure 4 above we show the region of the temperature-density plane where warm dense matter studies are important. Here we show the temperature $(T)$ in eV versus the density $(\square)$ in $\mathrm{g} / \mathrm{cm}^{3}$ both for hydrogen, a low $Z$ element, and aluminum, a moderate $Z$ element. The region where the theoretical uncertainties are largest are those where the standard theoretical approaches fail and 
experiments are exceedingly difficult. The difficulty arises theoretically from the fact that this is a regime where there are no obvious expansion parameters, as the usual perturbation expansions in small parameters used in plasma phase theories are no longer valid. Further, there becomes an increased importance on density-dependent effects, e.g., pressure ionization, as the surroundings starts to impinge on the internal structure of the ion or atom. Experimentally the study of warm dense matter is difficult, as the isolation of samples in this regime is complicated. Indeed, although the plasma evolution of every $[-T$ path that starts from the solid phase goes through this regime and plays an important role in its evolution, trying to isolate warm dense matter remains a major challenge.

It has been exceedingly difficult to perform experiments in the warm dense matter regime, which is, simply, why we know so little about it. As a first step, one must create a wellcharacterized warm dense matter state; the second is to gain information on the state through experiments. The first step has been the problem: warm dense matter is not a limiting case of matter, e.g., high- or low-temperature. When created in a laboratory environment, it does not tend to remain in a specified thermodynamic state for very long, making characterization difficult.

This WDM regime is accessed in all laboratory experiments where one creates a plasma from solid or near solid density targets; however, it is difficult to study this part of the plasma creation process in isolation. Rapid temporal variations, steep spatial gradients, and uncertain energy sources lead to indecipherable complexity. Indeed, although there has been much interest in this regime, witnessed by the literature on strongly coupled plasmas, there has been little progress. The interest generated in laboratory experiments is mirrored in the astrophysical literature where the warm dense matter regime is found, for example, in the structural formation of large planets and brown dwarfs. See ref. [13] for information on effects at 1 Mbar for hydrogen-bearing astrophysical objects; refs. [14-17], for information on Jovian planets; ref. [18]for extrasolar giant planets; refs. [19] and [20] for information on brown dwarfs; and ref. [21] for information on low mass stars.

The fact that the Heavy Ion beam source will allow the creation and probing of the warm dense matter regime in the laboratory, as discussed briefly below, will provide a set of data that will spark the field. The idea is simple but the impact may be vast, as the data obtained in the generation of the warm dense matter along an isochore, i.e., a track of constant density, with subsequent probing along the release isentrope, i.e., a track of constant entropy, will be unique and critically important for progress in the field. The importance of this data derives from the fact that to date the only possible method of generating warm dense matter is by shocking the material. The shock method provides information along the principal Hugoniot, that is, the locus of points in the pressure-density space that are accessed by a single shock - one point for each shock. Although this has been quite useful, it is a very limited set of data providing little information on the general behavior in the warm dense matter regime. Indeed, the amount of data that is currently available is so limited that one finds insufficient constraints on theoretical development. This can be illustrated by the curves in figure 2 where several predictions for an isochore of aluminum is presented in the temperature and pressure phase-space. Note that the four theories shown in the figure all predict theoretical Hugoniots that fit the experimentally determined Hugoniots, but all differ rather dramatically along the isochore. As aluminum is the most studied material, figure 2 can be interpreted as the minimum degree of uncertainty in this field of research and makes obvious the need for experimental data in this regime [24]. 


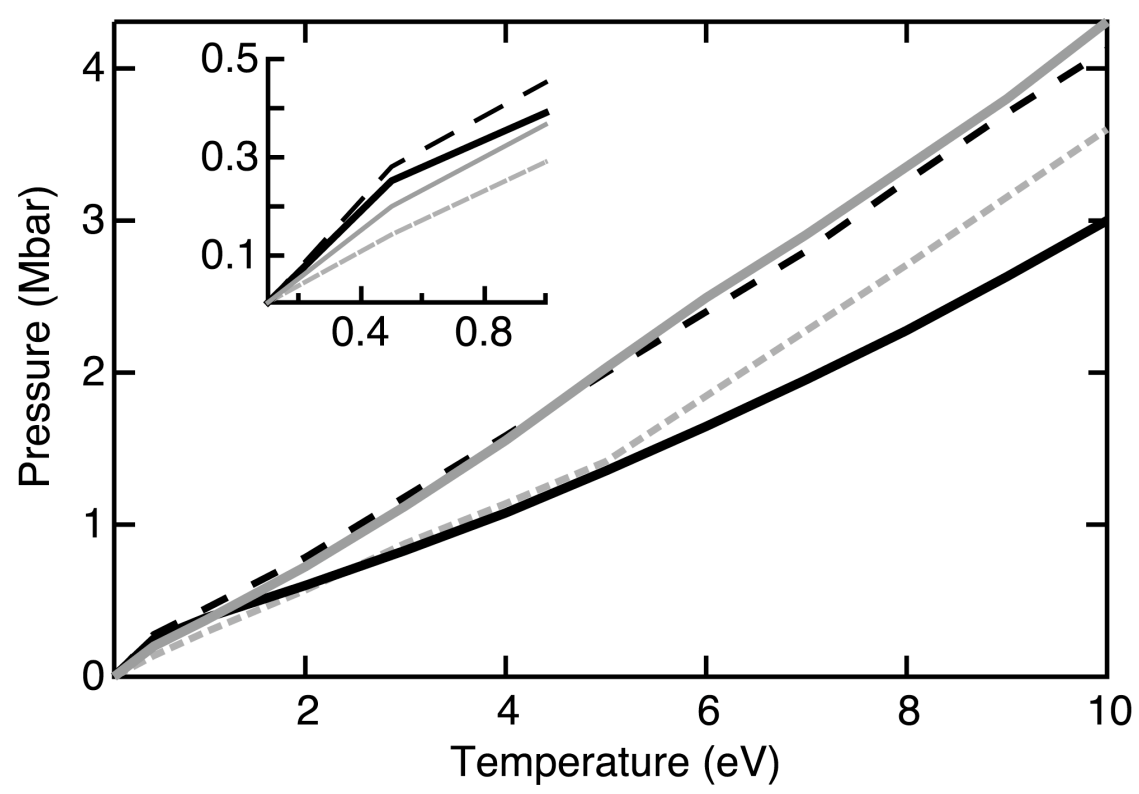

Figure 5. The isochore for aluminum in the warm dense matter regime for four theoretical models that all provide predictions consistent with the experimental points along the principal Hugoniot. The inset shows the low pressure low temperature region expanded. Data derived from heavy ion beam generated sources will assist in motivating theoretical developments for this important regime.

Further, one can illustrate the level of uncertainty in the equation of states by comparing models that are considered valid for simulations by virtue of the fact that where the data exist, i.e., along the principal Hugoniot, the models agree with experiment. In figure 6 we show two such examples. Here we have plot of the differences in the predicted pressure over a range of the temperature-density phase space that covers the warm dense matter regime for two elements, $\mathrm{Al}$ and $\mathrm{Cu}$. The choice of $\mathrm{Al}$ and $\mathrm{Cu}$ is pertinent as these are two of the most studied materials for shock-generated equations of state. In figure 3 the first thing to note is that there are substantial region within the warm dense matter phase-space where differences $>80 \%$ in the pressure are common. Second, we note that there are regions where the differences between the models are quite small and these correspond to those areas where the principal Hugoniot measurements have provided data to guide the theoretical development. Another way of saying this is that the measurements are essential for guidance and, indeed, this is what the heavy ion beam experimental capability will provide. 

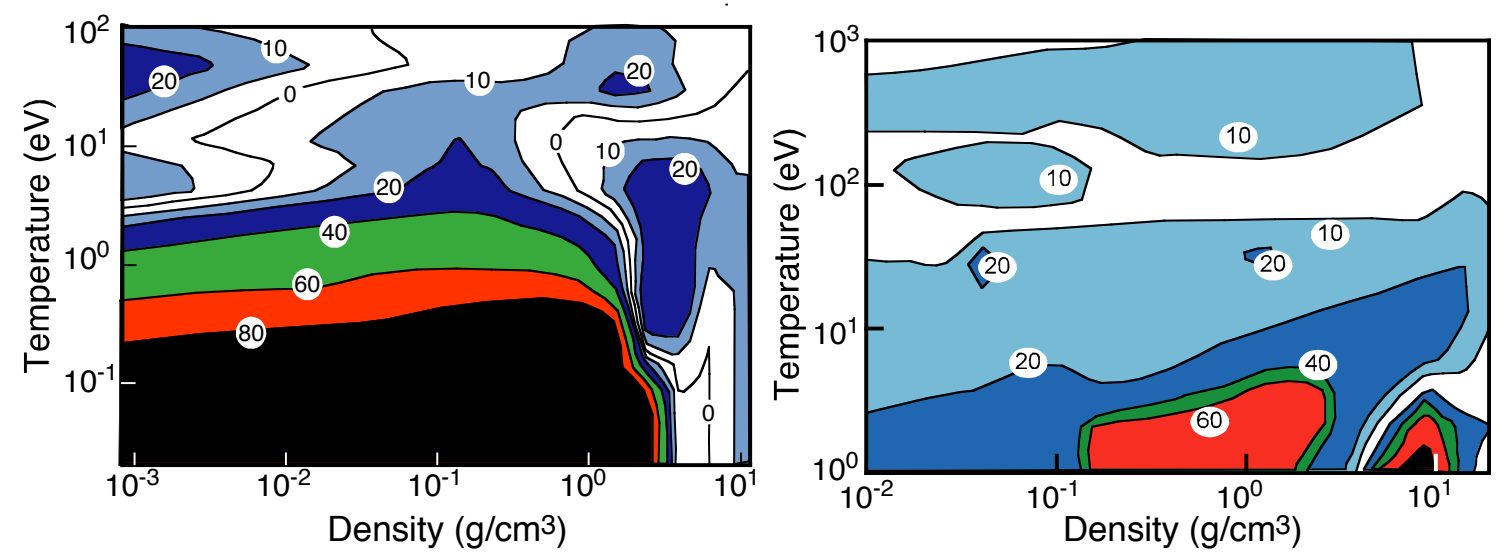

Figure 6. Comparison of the variation in pressure between two equations of state models commonly used, in the density-temperature phase space. Note that differences are greatest in the Warm Dense Matter regime.

\section{Targets and experiments for WDM studies:}

The design of the target will ultimately follow from the physics to be investigated. Some of the fundamental areas to be investigated include measurements of equation of state, thermal and electrical conductivity, and opacity. Other measurements will look at the transition of wire arrays or foams from the solid to the plasma state, examining the persistence of granularity and inhomogeneities in the system.

A number of types of measurements and diagnostics will be needed to obtain physical data on the state of the warm dense matter. These include:

1. Time dependent measurement of rarefaction waves and hydrodynamic expansion of the heated material, by imaging the emission.

2. Measurement of shock wave velocities, by imaging of emission and/or backlighting with x-rays, for those configurations in which shock waves are generated.

3. Measurement of the final charge state and energy of the ions after passing through the target. One can measure both total energy of the beam to determine the deposition and also the energy of the ions to further ensure that one understands the deposition mechanisms.

4. Measurement of the emission spectra of the heated target (in the WDM regime the temperatures are sufficiently low that at best VUV spectroscopy is needed - it is one of the charms of the regime that the medium is dense and there is little observable emission).

5. Measurement of the absorption spectra of the heated target being backlit by a laserproduced x-ray source.

6. Electrical resistivity measurements by applying a voltage across the target and observing the current response as the pulse is heated.

The targets themselves will have a variety of configurations. A simple planar target has been the basis for many of the calculations used for determining accelerator parameters. Typically, 
focal spot radii will be of order $1 \mathrm{~mm}$, and the range of medium mass ions in solid material in the few to tens of $\mathrm{MeV}$ energy range will be of order a few microns. So the aspect ratio of beam radius to target thickness will be $\sim 100$ to 1 , (i.e the targets will be flat and planar). This is in contrast to the experiments at GSI, where much larger ion energies ( $\sim 100$ 's of MeV/amu) are achieved with much lower currents $\left(\sim 10^{10}\right.$ ions/bunch, $\sim 200 \mathrm{~ns}$ pulse length), and so the target geometries tend to be long $(\sim 1 \mathrm{~cm})$ and cylindrical relative to the radius of $(\sim 1 \mathrm{~mm})$. The planar targets will tend to expand primarily longitudinally, in contrast to the predominantly radial expansion of cylindrical targets.

For lower density targets ( $\square$ as low as 0.01 times solid density) for the same ion range the physical length is inversely proportional to $\square$ so for the few to tens of $\mathrm{MeV}$ ions, the range can be 100 's of $\square \mathrm{m}$ up to a mm. In this case, the geometry of the heated region varies from thin up through the regime of near equality between longitudinal and transverse scales. Metal foams have been proposed for use on the National Ignition Facility (NIF) and for Inertial Fusion Energy (IFE) targets, so material testing of these components would be of benefit to both the Inertial Confinement Fusion (ICF) and IFE programs. Similarly, the evolution of wire arrays (as are being used on Sandia's Z-pinch machines) can be studied, and the transition from solid state to plasma state can be explored.

Other targets such as compressed gas targets, gas and liquid jets, and metal and other foams can be used to vary the density of the target over a wide range, so that a large fraction of the $\square-T$ plane can be explored using ion beam driven WDM.

The form of the experiments can be outlined simply. For example, the heavy ion beam can heat a sample isochorically. Then with measurements of the deposited energy, radiography of the heated volume, and in situ probes provided by high-energy laser generated x-rays one can determine the local volumetric expansion and temperatures. Thus the equation of state can be determined. Note in figure one that the area of phase space covered by a single experiment, although it maps out the points along the isentrope as expansion occurs, does not cover the entire space. Indeed, to cover the entire space one needs to make samples of less than solid density, e.g., underdense foams. In this manner one can span a large part of the interesting phase-space with changes of samples but with similar measurement techniques.

Finally, we note that although the experiment mentioned appears simple it is quite complex. First, the simultaneous measurement of a set of physical parameters in an experiment where all the data must be obtained on each shot necessitates the implementation of a number of diagnostics. This implementation, in turn, requires that the samples be constructed and have metrology performed to ensure that each diagnostic instrument (spectrometers, beam deposition, time resolved radiography, in situ scatter and/or absorption measurements) can obtain uncompromised data. This will, in turn, necessitate that shielding of the various components be ensured. Second, the accuracy required for equation of state measurements is highly dependent on the measurement of, for example, the expansion velocities that in turn is dependent on accurate distance versus time diagnostics. In those cases where one uses x-radiography to measure the expansion uniformity of the sample, alignment and diagnostic calibration (e.g., in a streak camera uncertainty in the sweep speed and its linearity) combine to make $10 \%$ accuracies difficult to attain. Third, the variation of the heavy ion beam focus, the variation in beam total energy, and the variation in the beam spectrum (here we mean velocity profile) requires that one have a series of reproducible experiments to evaluate a single data point in the EOS. Fourth, the need for reproducibility requires additional pulses. So repetition rates of order minutes or even 
seconds will be needed to account for the experiment preparations, calibrations, and accelerator variability.

\section{Estimating accelerator requirements}

In order to set the requirements on an ion accelerator for heating a target to Warm Dense Matter conditions, an understanding of the scaling of the energy loss rate $d E / d X$ is needed, where $E$ is the ion energy and $X \equiv \square \square d z$ is the integrated range of the ion. This quantity has been displayed graphically for a number of different ions, in ref [B1], and scaling to other target materials is also given.

For heating solid aluminum (at room temperature) over a range of ion mass from 4 amu (Helium) to $126 \mathrm{amu}$ (Iodine), the energy loss at the peak of the $d E / d X$ curve $\left(d E / d X_{\max }\right)$ may be parameterized approximately as:

$$
\left(1 / Z^{2}\right) d E / d X_{\max } \approx 1.09\left(\mathrm{MeVcm}^{2} / \mathrm{mg}\right) A^{-0.82}
$$

where $Z$ and $A$ are the ion nuclear charge and atomic mass, respectively. Expressing $d E / d X_{\max }$ as a function of A only yields:

$$
d E / d X_{\max } \approx 0.35\left(\mathrm{MeVcm}^{2} / \mathrm{mg}\right) A^{1.07}
$$

Thus, the peak energy loss rate increases (nearly linearly) with ion atomic mass.

Similarly, the energy at the peak increases with ion nearly quadratically with A:

$$
E\left(\text { at } d E / d X_{\max }\right) \approx 0.052 \mathrm{MeV} A^{1.803} \text {. }
$$

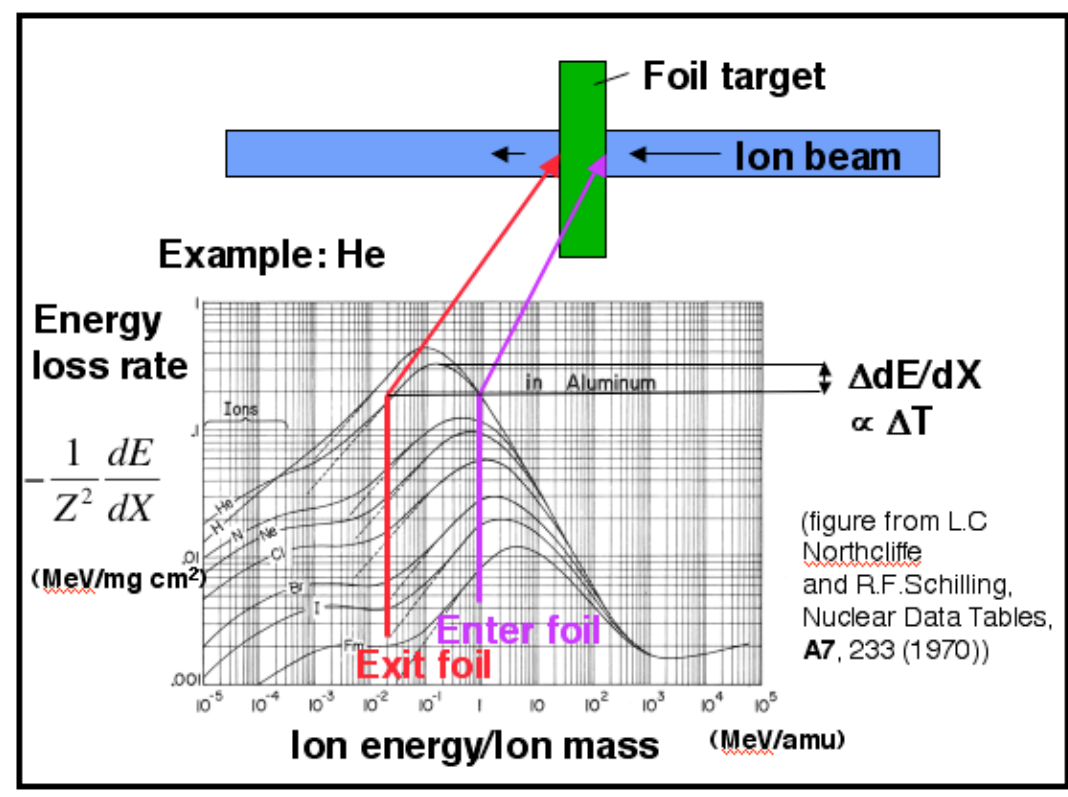

Figure 7. Temperature variations in an ion-beam heated foil can be minimized by choosing an ion and energy such that the peak in $\mathrm{dE} / \mathrm{dX}$ occurs in the center of the foil (ref. [B2]). 
Target uniformity is another important consideration. In ref. [6] it was pointed out that target temperature uniformity can be maximized in simple planar targets if the particle energy reaches the maximum in the energy loss rate $d E / d X$ when the particle has reached the center of the foil (see figure 7). For any specified fractional deviation in target temperature (assuming the energy is deposited in a time short so that no hydrodynamic, radiative, or other cooling has occurred) one can determine the energy at which the ion must enter and exit the foil. From the $d E / d X$ curves of ref. [26] we find that for the entrance and exit energies to have a 5\% lower energy loss rate relative to the peak in $d E / d X, \square \square / \square \approx 1.2$, where $\square E$ is the difference in ion energy between entering and exiting the foil, and $E$ is the energy at which $d E / d X$ is maximum. Note the large $(>1)$ fractional range in energy relative to peak energy is expected for a broad peak in a log-log representation. The spatial width of the foil $Z$, for a $5 \%$ temperature non-uniformity is then given by:

$$
Z=\square E /(\square d E / d X) \approx 0.77 \square A^{0.733}\left(\square_{a l} / \square\right)
$$

Here we have used $\square_{\mathrm{al}}=2.7 \mathrm{~g} / \mathrm{cm}^{3}$ to convert the range into a physical distance. So by using materials of low density such as metallic foams, for example, the width of the foil can be large, which can be advantageous as will be shown. The total energy density $U$, calculated from the total energy deposited over the course of the pulse and neglecting losses is thus:

$$
U=N_{\text {ions }} E / \square r^{2} Z=3.7 \times 10^{9}\left(\mathrm{~J} / \mathrm{m}^{3}\right)\left(N_{\text {ions }} / 10^{12}\right)(1 \mathrm{~mm} / r)^{2}\left(\square / \square_{\mathrm{al}}\right) A^{1.07}
$$

Here $N_{\text {ions }}$ is the number of ions in the pulse, and $r$ is the equivalent radius of the focal spot, defined such that the beam is assumed to have uniform density within $r$, and has zero intensity outside of $r$. So to achieve high energy density, large particle number, small spot radius, and higher target densities must be attained. In addition, to realize the energy density given by eq. (5), the hydrodynamic expansion timescale $Z / c_{s}$ must be much shorter than the pulse duration $\square t$.

\section{Hydrodynamic disassembly time:}

The sound speed $c_{s}$ is given by $c_{s}=\left([P / \square)^{1 / 2}=(\square \square 1] U / \square\right)^{1 / 2}$. Here $\square$ is the ratio of specific heats, $P$ is the pressure and $\square$ is the mass density. For estimating purposes, we take $\square$ to be $5 / 3$, although more refined estimates below will relax this assumption. For a "shock tube," that at a finite longitudinal distance $z$, has a discontinuous drop to zero pressure at some initial time, an analytical solution exists (ref. [27]; see fig. 8) in which a rarefaction wave propagates inward at speed $c_{s}$, and a plasma front flows outward at $2 c_{s}$. For the case of isochoric heating, when the pulse duration $\square t<<\square z / c_{s}$, where $\square z$ is the width of the foil, the dynamics will be the same as the shock tube solution. For times $\square t<\sim \square z / c_{s}$, we expect that, since the sound speed is increasing over the course of the pulse, the position of the rarefaction wave $z_{r}$ will be somewhat less than would be expected if calculated on the basis of the final heated plasma:

$$
z_{r}=\square_{0}^{t} s_{s} d t=\left.\frac{2}{3} c_{s^{*}} \square t \bigsqcup_{\square} t\right|^{12} .
$$

Here $c_{s^{*}}=c_{s}\left(T_{*}\right)$ and we assume $\frac{T}{T_{*}}=\frac{t}{\square t}$, where $\mathrm{T} *$ is the temperature achieved at the end of the ion pulse; we also assume $c_{s} \mu T^{1 / 2}$. 


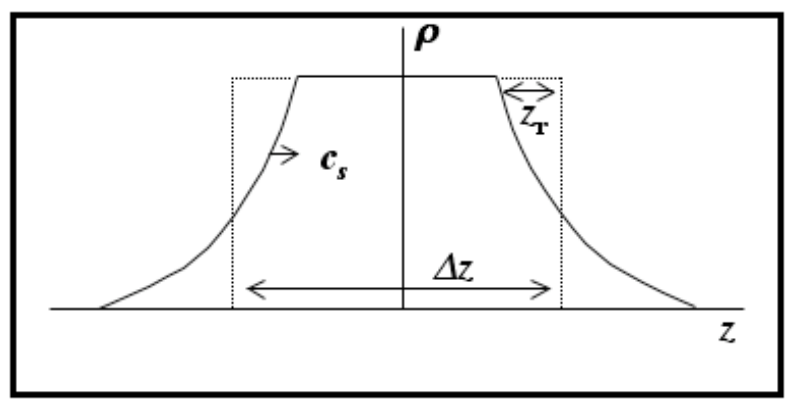

Figure 8. Schematic representation of rarefaction wave propagating inward at sound speed $c_{\mathrm{s}}$, and plasma front moving outward at speed $c_{\mathrm{s}}$. As material is heated over course of pulse $c_{\mathrm{s}}$ increases. The original density distribution of the foil is indicated by dotted line, and location of the rarefaction wave by $z_{\mathrm{r}}$.

We envision isochorically heating a target foil, and taking measurements with various optical or beam diagnostics. If our diagnostic is unable to resolve a volume smaller than the volume heated by the ion beam, and if we want to distinguish equations of state with $5 \%$ accuracy, then the sample volume cannot consist mostly of blow off material (i.e. material that is part of the rarefaction wave). If we demand that the blow off material is less than $5 \%$ of the total mass, that implies $2 z_{r} / \square z<0.05$, or

$$
\square t<3 \square z /\left(80 c_{s}^{*}\right)
$$

If on the other hand, the diagnostic has resolution $z_{\min }$ such that it can sample a fraction of the target $\left(z_{\min }<\square z\right)$, then, as long as the central part of the target has not been "contaminated" by the rarefaction wave, useful data can be obtained by just observing the central (heated) part of the foil. In this latter case, the pulse duration must satisfy

$$
\square t<3\left(\square z-\square z_{\text {min }}\right) /\left(4 c_{s} *\right) .
$$

If $\square z>>\square z_{\min }$, this can be a significantly longer time, but in any case, the longer of the two timescales above (eq. 7 and eq. 8) should be taken. For our examples to be discussed below, we have used $\square z_{\min }$ to be $40 \square$, which may be achievable using a $\mathrm{K}-\square$ diagnostic generated by a short pulse laser.

In order to calculate more accurately the sound speed, one needs to understand the response of the target to the energy deposited by the ion beam. In particular, the pressure and temperature will depend on the ionization state of the plasma. For our estimating purposes, we use a model developed by Zeldovich and Raizer and summarized in ref. [28]. The basic idea of the model is to calculate the average ionization state $Z^{*}$ by approximately solving the Saha equation and accounting for the ionization energy of each ion in the energy density $U$ (where $U$ $\left.=(3 / 2) n k T+Q\left(Z^{*}\right) \square / A m_{h}\right)$, and to include contributions to the pressure $P$ (where $P=n k T=k T$ $\left(Z^{*}+1\right) \square\left(A m_{h}\right)$ from the electrons and partially ionized target atoms. Here $Q\left(Z^{*}\right)=\square_{\mathrm{i}=1}^{Z^{*}} I_{\mathrm{i}}$, where $I_{\mathrm{i}}$ is the (known) ionization energy of the $\mathrm{i}^{\text {th }}$ level of the target material, $n$ is the total number density of ions, atoms, and electrons, and $\square$ is the mass density. Other more detailed equation of state models, including degenerate effects, correlation effects, and more exact treatment of the Saha equation, may have an impact on various transport and thermodynamic properties. These details are not to be minimized; after all that is why there is an experimental interest in this regime. For our purposes, however, the Zelodovich-Raizer equation of state allows approximate calculation of $Z^{*}$ (see fig. 9), $T$, and the coupling parameter $\square_{\mathrm{ii}}$. 


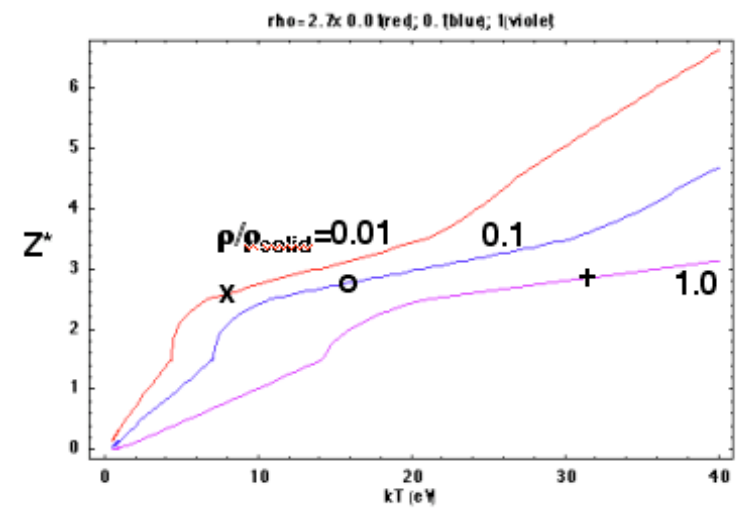

Figure 9. Calculation of ionization state, as a function of temperature for three different densities, using Zeldovich-Raizer equation of state. The $\mathrm{x}, \mathrm{o}$, and + would correspond to the conditions of reached in accelerator described by the central column of each of the $1 \%, 10 \%$, and $100 \%$ solid density cases in Table 2.

\section{Examples of accelerator requirements:}

Using the model described in the previous section for ion beam stopping, the time scale for hydrodynamic expansion and the equation of state we are able to make estimates of the required beam parameters for exploring the Warm Dense Matter regime. Tables 2 and 3 give examples of requirements for two different ion energy and mass, $\operatorname{Neon}^{+1}(A=20.17)$ at foil entrance energy $\left(E_{\max }\right)$ of $19 \mathrm{MeV}$, and Chlorine ${ }^{+1}(A=35.453)$ at $E_{\max }=52.4 \mathrm{MeV}$. The energy at the center of the foil $\left(E_{\text {center }}\right)$ and the energy at the exit of the foil $\left(E_{\min }\right)$ are listed in the captions to the tables. For each ion, three different mass densities of Aluminum target are given: Solid density $\left(2.7 \mathrm{~g} / \mathrm{cm}^{3}\right)$ and $10 \%$ and $1 \%$ of solid, which can be produced by making an aluminum "foam." In turn for each target density, three target temperatures are shown. Both tables are based on a minimum diagnosable length scale $Z_{\min }$ of $40 \square$. It is clear from the tables that solid density, although resulting in the highest energy density, requires vary short pulse durations, because the foil width is smaller than $Z_{\min }$ and so only a small rarefaction wave propagation distance is allowed. But for the $1 \%$ and $10 \%$ cases, the foil is larger than $Z_{\min }$, so that the rarefaction wave propagation distance can be 10 's or 100 's of microns, with concomitantly longer pulse duration times. In all cases the plasma temperature is in the few to tens of $\mathrm{eV}$, and the required number of particles is in the order of $10^{12}$ to $10^{13}$ particles, for equivalent focal spot radii of $1 \mathrm{~mm}$.

\begin{tabular}{|c|c|c|c|c|c|c|c|c|c|}
\hline \multirow{3}{*}{\begin{tabular}{|l}
$\mathrm{G}\left(\mathrm{g} / \mathrm{cm}^{3}\right)(\%$ solid $)$ \\
Foil length (m) \\
kT $(\mathrm{eV})$
\end{tabular}} & \multicolumn{3}{|c|}{$0.027(1 \%)$} & \multicolumn{3}{|c|}{$0.27(10 \%)$} & \multicolumn{3}{|c|}{$2.7(100 \%)$} \\
\hline & \multicolumn{3}{|c|}{700} & \multicolumn{3}{|c|}{70} & \multicolumn{3}{|c|}{7} \\
\hline & 3.5 & 7.9 & 15 & 4.5 & 15 & 20 & 7.1 & 31 & 38 \\
\hline Z* & 1.2 & 2.6 & 3.1 & 0.95 & 2.7 & 3 & 0.69 & 2.8 & 3.1 \\
\hline$\square_{\mathrm{ii}}=\mathrm{Z}^{*^{2}} \mathrm{e}^{<} \mathrm{n}_{\mathrm{i}}^{1 / 3} / \mathrm{kT}$ & 0.51 & 1 & 0.92 & 0.53 & 1.3 & 1.2 & 0.38 & 1.5 & 1.4 \\
\hline $\mathrm{N}_{\text {ions }} /\left(\mathrm{r}_{\text {spot }} / 1 \mathrm{~mm}\right)^{2} / 10^{12}$ & 2.24 & 7.96 & 22.4 & 2.24 & 14 & 22.4 & 2.24 & 22.4 & 30 \\
\hline bt (ns) & 56 & 30 & 18 & 2.5 & 1 & 0.8 & 0.03 & 0.01 & 0.008 \\
\hline $\mathrm{U}\left(\mathrm{J} / \mathrm{m}^{3}\right) / 10^{11}$ & 0.021 & 0.073 & 0.21 & 0.21 & 1.27 & 2.1 & 2.1 & 21 & 28 \\
\hline
\end{tabular}

Table 2. Neon beam: $Z=10, A=20.17, E_{\min }=4.4 \mathrm{MeV}, E_{\text {center }}=11.7 \mathrm{MeV}, E_{\max }=19 \mathrm{MeV}$, and $\square \mathrm{z}_{\min }=40 \square$ 


\begin{tabular}{|l|c|c|c|c|c|c|c|c|c|c|}
\hline \hline$\left(\mathrm{g} / \mathrm{cm}^{3}\right)$ (\%solid) & \multicolumn{3}{|c|}{$027(1 \%)$} & \multicolumn{3}{c|}{$0.27(10 \%)$} & \multicolumn{3}{c|}{$2.7(100 \%)$} \\
\hline Foil length (m) & \multicolumn{3}{|c|}{1050} & \multicolumn{3}{c|}{105} & \multicolumn{3}{c|}{10.5} \\
\cline { 2 - 10 } $\mathrm{kT}(\mathrm{eV})$ & 3.5 & 7.9 & 15 & 4.6 & 15 & 20 & 7.1 & 31 & 38 \\
$\mathrm{Z} *$ & 1.2 & 2.6 & 3.1 & 0.96 & 2.7 & 3 & 0.69 & 2.8 & 3.1 \\
$\mathrm{Z}_{\mathrm{ii}}=\mathrm{Z}^{2} \mathrm{e}^{2} \mathrm{n}_{\mathrm{i}}{ }^{1 / 3} / \mathrm{kT}$ & 0.51 & 1 & 0.76 & 0.53 & 1.3 & 1.1 & 0.38 & 1.5 & 1.4 \\
$\mathrm{~N}_{\text {ions }} /\left(\mathrm{r}_{\text {spot }} / 1 \mathrm{~mm}\right)^{2} / 10^{12}$ & 1.24 & 4.3 & 12.4 & 1.24 & 8 & 12.4 & 1.24 & 12.4 & 16 \\
$\mathrm{at}(\mathrm{ns})$ & 87 & 46 & 27 & 5.6 & 2.2 & 1.8 & 0.045 & 0.014 & 0.012 \\
$\mathrm{U}\left(\mathrm{J} / \mathrm{m}^{3}\right) / 10^{11}$ & 0.021 & 0.073 & 0.21 & 0.21 & 1.35 & 2.1 & 2.1 & 21 & 28 \\
\hline
\end{tabular}

Table 3. Chlorine beam: $Z=17, A=35.453, E_{\min }=12.3 \mathrm{MeV}, E_{\text {center }}=32.4 \mathrm{MeV}, E_{\max }=52.4 \mathrm{MeV}$, and $\square \mathrm{z}_{\min }=40 \square$.

\section{Tolerance on Velocity Spread:}

Several different types of accelerators are being considered to produce the very short $(<\sim$ ns) pulses required for HEDP studies. But one common thread in all of the approaches, has been the need to invoke neutralized drift compression, to overcome the limit imposed by spacecharge. Neutralized drift compression is a departure from the more traditional approach of nonneutral drift compression that allows the longitudinal space charge to cause the beam velocity to "stagnate," thereby removing the velocity tilt, just as the beam is passing through the final focusing magnets, thus minimizing any potential chromatic aberrations that arise in the final focusing process. Using neutralized drift compressions achieves shorter pulses, but the various longitudinal parts of the beam that have different longitudinal velocities maintain those velocities through to the end, including the final focus. So, not only do the final focusing optics have to be tolerant of velocity spread, but target heating uniformity must be maintained as different parts of the beam (with different longitudinal velocities) will have different stopping powers $(d E / d X)$ and which in principal lead to a temperature variation larger than that of a single particle near the Bragg peak.

To investigate the effect of velocity spread we integrated the $d E / d X$ curves of ref. [26]. As an example we investigated the evolution of a He ion beam propagating through $1 \square$ foil of aluminum (see figs. 10 and 11). To represent the effect of a velocity spread we chose a number of different ion energies and averaged the energy loss rate at each point in the foil (corresponding to a energy distribution that is uniform between an lower and upper energy cutoff), and then calculated the maximum change in energy loss rate and normalized to the average energy loss rate in the foil $(=\square T / T)$. In the $1 \square$ foil case, for Helium with energy centered about $0.8 \mathrm{MeV}$ and with zero energy spread, there was a $1.8 \%$ fractional spread in $d E / d X$ through the foil. (So $\square T / T=0.018$ for this example, and is defined as the difference between the maximum and minimum energy loss rate divided by the average energy loss rate). As we increased the energy spread of the He beam, the calculated $\square T / T$ did not significantly increase until the velocity spread $\square v_{\text {spread }} / v=(1 / 2) \square E_{\text {spread }} / E$ is of order the fractional energy change of a single particle through the foil $\square E_{\text {single_particle }} / E$. Here $\square E_{\text {spread }}$ is the half width of the uniform particle distribution in energy and $\square v_{\text {spread }}$ is the corresponding velocity spread. The general conclusion, would appear to be that if $\square E_{\text {spread }}<\sim \square E_{\text {single } \text { particle }}$ then there is no appreciable degradation of the uniformity. On the other hand, there does not appear to be a significant advantage in a small but finite energy spread. Both statements need to be verified over a broad range of foil thickness and particle energy spreads, and the dependence on particle distribution function needs to be explored. If confirmed the temperature uniformity variations in the target may not be the most severe 
limitation to the allowed energy spread from velocity tilt, but more likely final optics considerations.
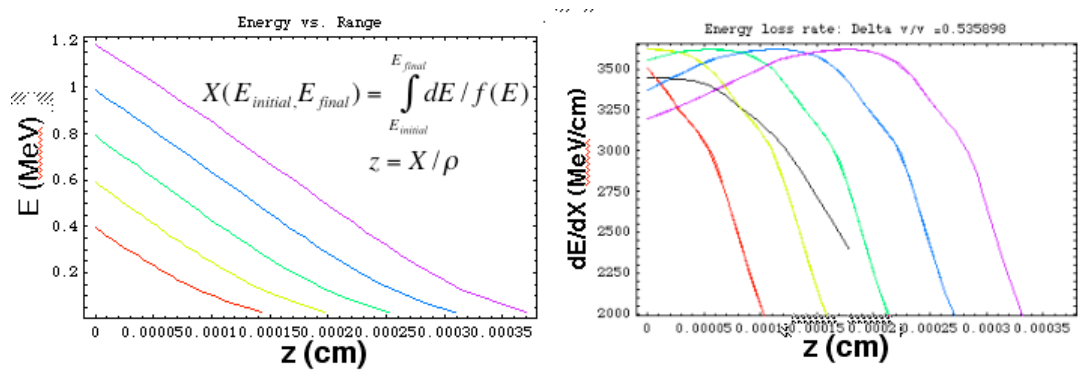

Figure 10. Energy vs. distance and $\mathrm{dE} / \mathrm{dX}$ vs distance, for a He ion propagating in cold aluminum, for five different energies ranging from 0.4 to $1.2 \mathrm{MeV}$. The black curve in the right hand figure is the average of the five colored curves and represents the total average energy loss rate for an ion distribution function that is uniform in energy.

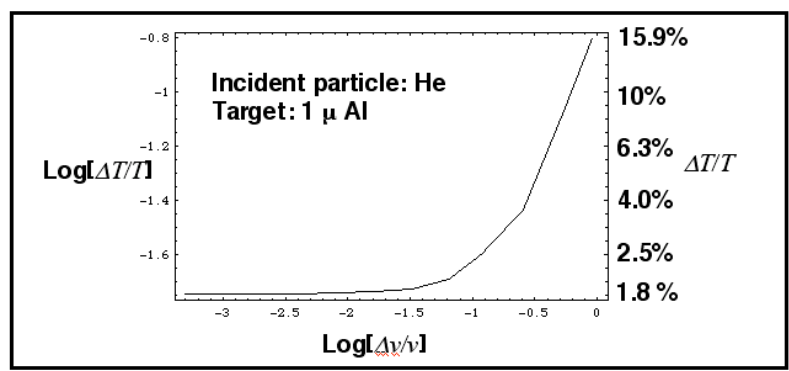

Figure 11. Temperature uniformity vs. velocity spread, for a He beam with central energy $0.8 \mathrm{MeV}$, propagating through a $1 \square$ cold aluminum foil.

Not only do HEDP experiments require uniform deposition but they also require high intensity, which means both short pulse and small beam radius. We may make simple estimates for the contribution to the spot size from chromatic effects (i.e. for the effects of a velocity spread) from a number of optical systems. For example, for a "thick" solenoidal lens in which a beam enters a solenoid with zero convergence angle and focuses to a spot within the solenoid, it can be shown to have a radius from emittance and chromatic effects $r_{\text {spot }}$ to be approximately:

$$
r_{\text {spot }}^{2} \square\left(\square r_{0} / 2\right)^{2}\left(\square v_{\text {spread }} / v\right)^{2}+\left(2 \square f / \square r_{0}\right)^{2}
$$

where $r_{0}$ is the radius of the beam at the entrance to the solenoid, $f$ is the focal length, i.e., the distance from the entrance of the solenoid to the focal spot, and $\square$ is the beam emittance. The quantity $r_{\text {spot }}$ is minimum when $r_{0}^{2}=(2 / \square \square] /\left(\square v_{\text {spread }} / v\right)$ and has the value

$$
r_{\text {spot }}^{2}=2 \square \square v_{\text {spread }} / v
$$

At minimum pulse duration the velocity tilt is converted to a velocity spread, so achieving high beam intensity will limit the velocity tilt. A system which is less sensitive to velocity tilt has also been proposed, such as the adiabatic plasma lens, but the dynamic range of these types of lens are generally limited to a reduction in spot size to a factor of around 2 or less, so these will most 
likely be used as a final "after burner" optic, with the bulk of the focusing being carried out by a conventional, solenoid optic, for which equations (9) and (10) provide limits.

\section{Conclusions}

Accelerator-produced heavy ion beams offer unique capabilities to drive matter to the densities and temperatures known as the High Energy Density Physics regime (and particularly that part of the parameter space known as the Warm Dense Matter regime). Considerations of the precise control and uniformity of energy deposition, relatively large sample sizes, a benign diagnostic environment, high shot rates with multiple beamlines, and the possibility of easy access by a broad range of investigators all point to a useful and unique role for heavy ion accelerators to play in exploring the physics of this regime. Further, a plan of near term accelerator research studying a new method of reaching high intensity, i.e. neutralized drift compression followed by neutralized focusing, is the enabling physics that would lead to a user facility within a 10-year timescale. We are beginning to lay the ground work for the optimization of such a facility, by considering a range of options (ion mass and energy, acceleration method, target and diagnostic configurations). This work has just begun but it is our conviction that the scientific discovery potential of such a facility would be high, and if the facility were built it could be the "spark" that ignites the field.

\section{Acknowledgements}

The authors would like to thank Dick Briggs, Debbie Callahan, Chris Celata, Andy Faltens, Alex Friedman, Larry Grisham, Enrique Henestroza, Ed Lee, Jay Marx, Lou Reginato, Dave Rose, Andy Sessler, John Staples, Max Tabak, Will Waldron, Dale Welch, Jonathan Wurtele, Simon $\mathrm{Yu}$ for their ideas, discussions, calculations and simulations that have contributed to our current understanding of ion-driven HEDP.

\section{References}

1. M Turner, et.al., Connecting Quarks with the Cosmos: Eleven Science Questions for The New Century (National Academies Press, 2003).

2. R. C. Davidson, et. al., Frontiers in High Energy Density Physics - The X-Games of Contemporary Science (National Academies Press, 2003).

3. The Science and Applications of Ultrafast, Ultraintense Lasers: Opportunities in Science and Technology Using the Brightest Light Known to Man (Report on the SAUUL Workshop, June 17-19, 2002).

4. R. C. Davidson, et. al., Frontiers for Discovery in High Energy Density Physics, (prepared by the National Task Force on High Energy Density Physics July 7, 2004)

5. An Integrated High Energy Density Physics Program at LBNL Based in the Office of Science LBNL White Paper sent by LBNL Director Charles Shanks to DOE Science Director Ray Orbach September 1, 2003

6. L. R. Grisham, Moderate Energy Ions for High Energy Density Physics Experiments, Physics of Plasmas, in press (2004).

7. D. Varentsov, editor, "Studies on High Energy Density Matter with Intense Heavy Ion and Laser Beams a GSI" (HEDgeHOB), a white paper on proposed collaboration in warm dense matter research at GSI April 13, 2004 
8. "Implementing Agreement between the Department of Energy of the United States of America and the Federal Ministry of Education and Research of the Federal Republic of Germany on Collaboration in the Field of Dense Plasma Physics" (DOE Office of Fusion Energy Science, September 2001)

9. S.S. Yu, et al., Nuclear Instruments and Methods in Physics Research A, in press (2004).

10. D.R. Welch, et al., Nuclear Instruments and Methods in Physics Research A, in press (2004).

11. M. Leitner, et al., Nuclear Instruments and Methods in Physics Research A, in press (2004).

12. N. Tahir, et al., Nuclear Instruments and Methods in Physics Research A, in press (2004).

13. Van Horn, H. M. (1991). Science 252, 384-389.

14. Smoluchowski, R. (1967). Nature 215, 691-695.

15. Hubbard, W. B. (1981). Science 214, 145-149.

16. Chabrier, G., Saumon, D. Hubbard,W. B. \& Lunine, J. I. (1992). Ap. J. 391, 817-826.

17. Nellis, W. J., Ross, M.\& Holmes, N. C. (1995). Science 269, 1249-1252.

18. Saumon,D., Hubbard, W. B., Burrows, A., Guillot, T., Lunine, J. I. \& G. Chabrier (1996)Ap. J. 460, 993-1018.

19. Saumon,D., Hubbard, W. B., Chabrier, G. \&Van Horn, H. M. (1992). Ap. J 391, 827831.

20. Hubbard, W. B., Guillot, T., Lunine, J. I., Burrows, A., Saumon, D., Marley, M. S. \& Freedman, R. S. (1997). Phys. Plasmas 4, 2011-2015.

21. Chabrier, G. \& Baraffe, I. (1997). Astron. Astrophys. 327, 1039-1053

22. Huba, J. D. (2000). NRL Plasma Formulary (Office of Naval Research. Washington. DC) http://wwwppd.nrl.navy.mil/nrlformulary/nrlformulary.html.

23. More, R. M., Warren, K.H., Young, D.A.. \& Zimmerman, G.B. (1988). Phys. Fluids, 31, 3059-3078.

24. Rogers, F. J. \& Young, D. A. (1997). Phys. Rev. E 56, 5876-5883.

25. Rogers, F. J. (2000).Phys. of Plasmas 7, 51-5.

26. I.C. Northcliffe and R. F. Schilling, "Range and Stopping Power Tables for Heavy Ions," Nuclear Data Tables, A7 233-463 (1970).

27. L.D. Landau and E.M. Lifshitz, Fluid Mechanics, [Pergamon Press, Oxford], Chapter 10, Section 92, problem 2. (1959).

28. R. J. Harrach and F.J. Rogers, J. Appl. Phys. 52, 5592 (1981). 
Appendix 1. The heavy-ion section (Section 3.2), from the National Task Force report [4]

\subsection{Heavy-Ion-Driven High Energy Density Physics and Fusion}

Accelerators producing appropriately tailored energies of intense heavy ion beams can provide a useful tool for creating uniform high energy density matter to study the stronglycoupled plasma physics of warm dense matter in the near term, and for inertial fusion in the longer term. Both fusion and high energy density physics applications of heavy ion beams require understanding the fundamental physics limits to the compression of ion beams in both space and time before they reach the target, as well as a basic understanding of collective beamplasma interaction processes and beam energy deposition profiles within the dense plasma targets. This thrust area focuses on the beam and target physics knowledge base needed over the next ten years for future heavy ion beam applications to high energy density physics and fusion. The emphasis during the first five years is on determining the physics limits to heavy ion beam longitudinal compression and transverse focusing upstream of the target, and during the second five year period, an increased effort is planned for beam-target interaction physics and target diagnostic development for high energy density physics. This heavy ion high energy density physics thrust would also make significant contributions towards heavy-ion-driven inertial fusion.

\subsubsection{Motivating Intellectual Question}

How can heavy ion beams be compressed to the high intensities required for creating high energy density matter and fusion ignition conditions?

Heavy ion beams have a number of advantages as drivers of targets for high energy density physics and fusion. First, heavy ions have a range exceeding the mean-free-path of thermal x-rays, so that they can penetrate and deposit most of their energy deep inside the targets. Second, the range of heavy ion beams in dense plasma targets is determined primarily by Coulomb collisions with the target electrons. The ions slow down with minimal side-scattering, and their energy deposition has a pronounced peak in the rate of energy loss $\mathrm{dE} / \mathrm{dx}$ that increases with the beam ion charge state $Z$. These properties make heavy ions an excellent candidate for high energy density physics studies, where thin target plasmas would be uniformly heated by locating the deposition peak near the target center. The primary scientific challenge in exploiting these desirable properties in the creation of high energy density matter and fusion ignition conditions in the laboratory is to compress the beam in time (by 1000 times overall, requiring 10100 times more longitudinal bunch compression than present state-of-the-art) to a pulse length that is short compared to the target disassembly time, while also compressing the beam in the transverse direction (by 10 times) to a small focal spot size for high local deposition energy density. Proposed new experiments compressing intense ion beams within neutralizing plasma would significantly extend the beam current into high-intensity regimes where the beam would not otherwise propagate in the absence of background plasma, and where beam-plasma collective effects with longitudinal and azimuthal magnetic focusing fields have not been previously explored. 
A basic understanding of the collective processes and nonlinear dynamics of intense, high-brightness, heavy ion beams, and a determination of how best to create, accelerate, transport, compress and focus these beams to a small spot size are critical to achieving the scientific objectives of heavy ion fusion and ion-beam-driven studies of warm dense matter. Most of the kinetic energy of heavy-ion beams is in the directed motion of the beam particles, but a small fraction is in random kinetic energy, characterized by the effective temperature of the beam particles. Plasma electrons can be used to neutralize much of the repulsive space charge that resists the beam compression in time and space, but the beam temperature ultimately limits the smallest achievable spot size and pulse duration after the space charge forces are removed from the beam inside plasmas. To minimize the beam temperature, and thereby maximize the energy deposition in the target, the beam dynamics must be controlled with high precision throughout the entire dynamical trajectory, using accurately positioned and tuned confining magnets, carefully tailored accelerating fields, and final charge neutralization techniques that do not degrade the beam quality.

There are key synergistic relationships of the research on intense heavy ion beams to understanding the nonlinear dynamics of intense charged particle beams for high energy and nuclear physics applications, including minimization of the deleterious effects of collective processes such as the two-stream (electron cloud) instability, and the use of a charge-neutralizing background plasma to assist in focusing intense beams to a small focal spot size (plasma lens effect).

\subsubsection{Research Opportunities}

Target and Accelerator Requirements: A recent sub-panel of the Fusion Energy Sciences Advisory Committee [1] reports "Inertial fusion energy capabilities [laser, accelerator and z-pinch drivers for fusion energy] have the potential for significantly contributing to high energy density physics and other areas of science. For example, isochoric heating of substantial volumes to uniform, elevated temperatures should be achievable using heavy ion beams... Moreover, the rapid turnaround capabilities envisioned for inertial fusion energy drivers could accelerate progress in HEDP science by enabling a wide community of users to conduct "shot-on-demand" experiments with data rates and volumes far exceeding those obtained on large systems that currently require long times between shots." A s indicated by the scientific question and supporting narrative for heavy-ion-driven high energy density physics and fusion, the primary scientific challenge is to compress intense ion beams in time and space sufficiently to heat targets to the desired temperatures with pulse durations of order or less than the target hydrodynamic expansion time. For low energy ions (in the few to tens of $\mathrm{MeV}$ range), requirements to study strongly-coupled plasma properties in the warm dense matter regime are: target foils of thickness a few to tens of microns, 1 to 20 Joules (in a single beam), 0.5 to $10 \mathrm{eV}$ temperature, 0.2 to 2 nanosecond final pulse duration, and 0.5 to $2 \mathrm{~mm}$ diameter focal spot size. Target diagnostics for high energy density physics studies should have spatial resolution small compared to the focal spot size, temporal resolution small compared to the target hydrodynamic expansion time after heating, and energy deposition measurement accuracy better than 3\%. For x-ray production in inside indirect-drive fusion targets, ion beams must heat foam layers $1-100 \%$ that of solid-density with 50 to $200 \mathrm{~kJ}$ per beam (many beams), $200 \mathrm{eV}$ target radiation temperature, 5 to 10 nanosecond final pulse duration, and 4 to $10 \mathrm{~mm}$ diameter focal spot size. For high energy density physics studies, ranges of ions with 0.2 to 1 $\mathrm{MeV} / \mathrm{u}$ should be larger than the target thickness, with the deposition peak centered in the target 
in order to achieve maximum uniformity inside the target for accurate measurements of the heated plasma properties, and to allow analysis of transmitted ion energies and charge states as a diagnostic. Hydrodynamic codes with a capability for calculating energy deposition from a distribution of incident ion energies and angles should evaluate changes in observable target properties for different equation-of-state models. For fusion, radiation transport is a key additional target code capability that is required. Ion ranges with 10 to $20 \mathrm{MeV} / \mathrm{u}$ should be less than the target radiator thickness, but larger than the mean free path of the target x-rays so that the peak ion deposition can occur inside the radiation case (hohlraum) surrounding the fusion fuel capsule.

The minimum pulse length and focal spot radius depend on the final longitudinal and transverse effective temperatures, respectively, accumulated from all non-ideal effects experienced by the ion beam as it travels from the source through the accelerator, and through longitudinal compression and final focus onto the target. Accelerators for both high energy density physics and fusion must initially inject sufficiently bright (low temperature) beams, accelerate the heavy ions to the desired energy range, and then longitudinally compress and radially focus the beams onto the target with minimal growth in the longitudinal beam temperature (much less than a factor of 10 to allow overall axial bunch compression by a factor of 100 or more), and with minimum transverse temperature growth (much less than a factor of 10 to allow radial focusing by more than a factor of 10 ).

Scientific Objectives and Milestones: $\quad$ Advances over the past several years include: (i) high current ion sources and injectors ( 0.1 to $1 \mathrm{~A}$ of potassium) have been shown to have adequate initial beam brightness (sufficiently low transverse and parallel temperatures) to meet the above requirements at injection; (ii) negligible beam brightness degradation has been observed in transport of $200 \mathrm{~mA}$ potassium ion beams through electric quadrupole focusing magnets; and (iii) more than $95 \%$ of potassium beam space charge has been neutralized with preformed plasma over $\sim 1$ meter lengths without deleterious beam-plasma instabilities. Over the next five years, before beam-on-target experiments begin, the research will address the key remaining beam physics issues necessary to meet the accelerator requirements described above. These fall into four scientific areas:

(1) High brightness heavy ion beam transport in magnets, particularly to understand limits on beam-channel wall clearance (aperture fill) imposed by gas and electron cloud effects, together with beam matching and magnet non-linearities.

(2) Longitudinal compression of intense ion beams, particularly to understand limits on longitudinal compression within neutralizing background plasma, and the effects of potential beam-plasma instabilities over distances longer than 1 meter.

(3) Transverse focusing onto targets, particularly to understand limits on focal spot size set by chromatic aberrations due to uncompensated velocity spreads from upstream longitudinal compression, and beam temperature growth from imperfect charge neutralization.

(4) Advanced beam theory and simulation, particularly developing, optimizing and validating multi-species beam transport codes that can predict self-consistently the beam loss with gas and electron clouds, and developing integrated beam simulation models required to analyze source-to-target beam brightness (temperature) evolution.

After the beam physics issues identified above are favorably addressed over the next five years, emphasis will be placed on the fifth scientific thrust area: 
(5) Beam-target interactions, particularly to understand beam deposition profiles within thin foil targets and the potential uniformity of isochoric heating, accounting for target and beam ion charge state conditions, including development of accurate beam deposition and lasergenerated x-ray target diagnostics, and extension of integrated beam simulation models from source through target.

These scientific areas will be pursued with an overall 10-year objective of providing the beam and target physics knowledge base for a future $\sim \$ 50 \mathrm{M}$-class heavy-ion accelerator-based high energy density physics facility for achieving 1-10 eV solid-density plasmas by isochoric ion heating with uniformity and diagnostic resolution adequate to discriminate the predictions of various ab initio theories for strongly-coupled plasmas. Successful achievement of this objective will address the Office of Management and Budget/Office of Fusion Energy Sciences 10-year measure for inertial fusion energy/high energy density physics: "With the help of experimentally validated theoretical and computer models, determine the physics limits that constrain the use of inertial fusion energy drivers in future key integrated experiments needed to resolve the scientific issues for inertial fusion energy and high energy density physics". In addition, such an accelerator-driven high energy density physics facility would represent an important step towards the long-term objective of heavy-ion-driven inertial fusion.

Research Tools, Facility Requirements, and Milestones: Several specific facility requirements with intermediate two-year and five-year milestones (for experiments and modeling) are required to measure progress towards the 10-year objective. These include:

\section{Two-Year Science Goals (FY06):}

A 2: Intermediate experiments to assess the physics limits of neutralized ion beam compression to short pulses. Measure the parallel and transverse temperature of a high perveance ion beam (space-charge potential / kinetic energy larger than $10^{-4}$ ) before and after longitudinal compression by a factor of ten in neutralizing background plasma, and before and after pre-bunching of initially non-neutral ion beam in an acceleration-deceleration system. This series of experiments and modeling is needed to design integrated experiments combining neutralized drift compression and final focusing.

B2: Intermediate experiments to develop a predictive capability for gas and electron effects. Compare measured and calculated effects of gas and electron clouds on beam temperature as a function of beam aperture fill factors initially in transport lines with four magnets (quadrupoles and solenoids). This series of experiments and modeling will provide the scientific basis for future experimental upgrades.

\section{Five-Year Science Goals (FY09):}

A5: Integrated beam experiments on neutralized compression and focusing onto targets. Compare the measured and simulated focal spot beam intensity profiles in integrated experiments with beam current and energy upgraded from that used in A2, with a goal of $1 \mathrm{eV}$ temperature in targets (a temperature corresponding to the high energy density threshold level of $10^{11} \mathrm{~J} / \mathrm{m}^{3}$ at solid density). This series of experiments and modeling of compression and 
focusing will provide the physics basis for a future heavy-ion high energy density physics facility.

B5: Demonstrate predictive capability for gas and electron effects for a heavy-ion high energy density physics facility. Compare measured and calculated effects of gas and electron clouds, in combination with beam matching and magnet errors, assuming B2 results warrant an upgrade to longer lattice transport experiments. This series of experiments and modeling is essential to determine the magnet apertures of quadrupole and solenoid transport options for a future heavyion high energy density physics facility.

Figure 3.1 gives a timeline with milestones and resource requirements.

Opportunities for Interagency Cooperation: Several opportunities exist for scientific cooperation between the heavy-ion-driven high energy density physics/fusion thrust area sponsored by the Office of Fusion Energy Sciences (OFES) and other federal agencies. These include:

1. Office of Basic Energy Sciences (OBES), with the Spallation Neutron Source (SNS) at the Oak Ridge National Laboratory, in common areas of need for data on wall secondary electron production and gas desorption induced by beam loss [2], and in multi-species particle-in-cell simulation models of the impact of gas and electron clouds on the beam, including two steam instabilities [3,4]. This area may be critical to the achievement of full average beam power and neutron production in the SNS.

2. National Nuclear Security Agency (NNSA), with the Proton Storage Ring (PSR) and Dual Axis Radiographic Hydro Test facility (DARHT) at the Los Alamos National Laboratory [3, 4], in common areas of modeling multi-species gas/electron effects including two-stream instabilities (PSR), and in efficient computational techniques with multi-species modeling of electron beam neutralization from gas and ions backstreaming from the targets (DARHT).

3. Collaborations with the high energy and nuclear physics accelerator communities on joint development of advanced computational tools are important to predict and control electron cloud effects, beam halo production and associated losses, including use of Adaptive Mesh Refinement techniques [5] and nonlinear perturbative ( $\square$ f) particle simulation techniques [3] developed for modeling heavy ion experiments. Sharing these computational tools can greatly increase the range of intense beam physics problems that can be modeled for a variety of scientific applications.

4. Within strongly-coupled plasma regimes of high energy density physics, scientific progress would benefit from comparisons of equation of state and constitutive properties data obtained using heavy ion isochoric heating with similar data obtained using other future high energy density physics drivers, including lasers, Z-pinches, and X-ray free electron lasers (XFELs)[6]. 
Figure 3.1: Timeline and Resource Requirements for Heavy-Ion Driven HEDP/fusion

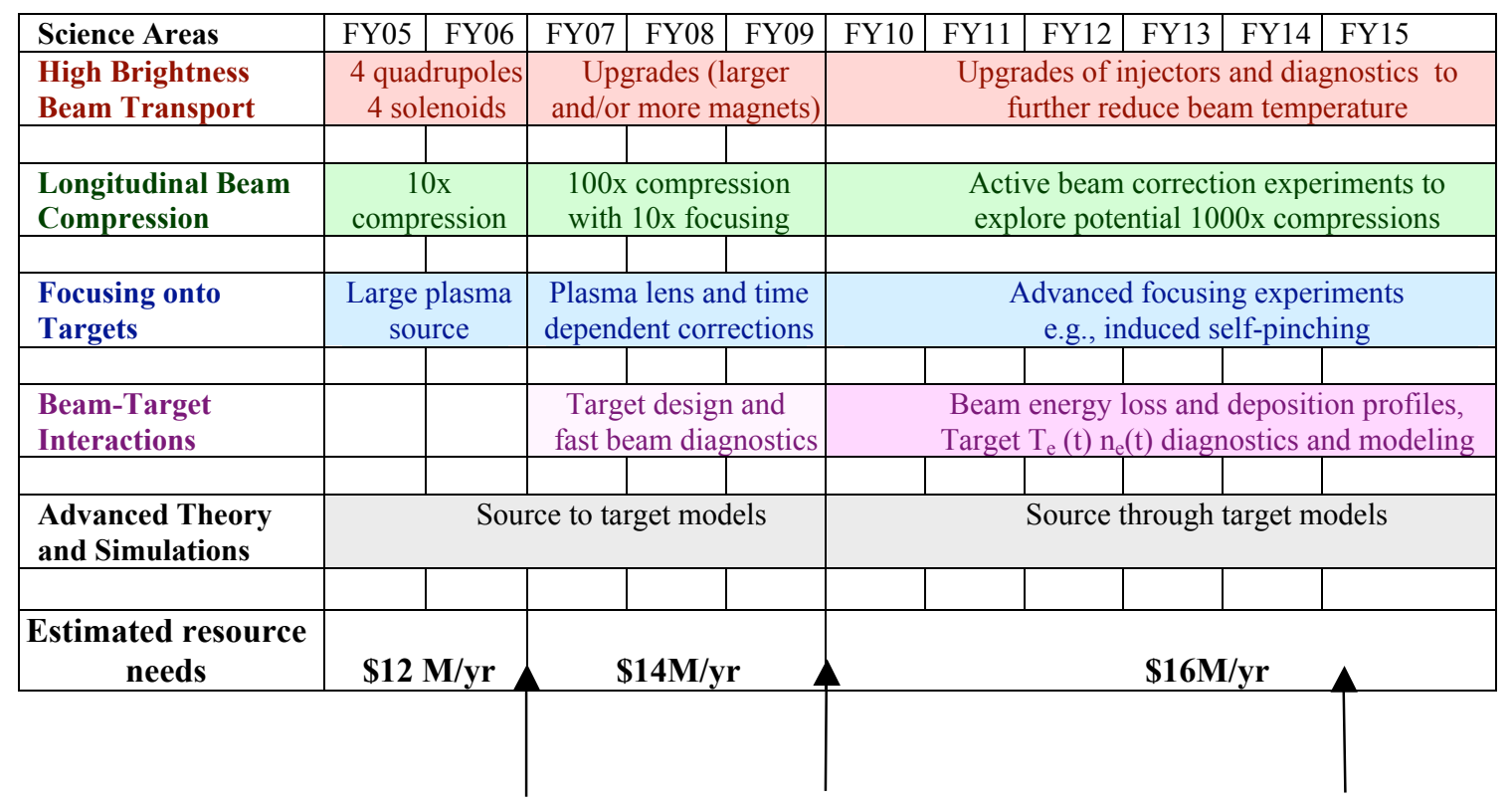

\author{
10x neutralized \\ compression \\ Gas/electron limits \\ in 4 magnets
}

\author{
5yr Milestones \\ 100x neutralized compression and \\ focusing \\ Gas/electron predictive capability \\ for HEDP accelerators
}

\author{
10yr Objective: \\ Beam and target physics \\ knowledge base for \\ heavy-ion-driven HEDP \\ user facility
}

\section{References}

[1] “A Review of the Inertial Fusion Energy Program” Final Report to FESAC, March 29, 2004

[2] A.W. Molvik, et al., "Electron effects in intense, ion beam linacs theory and experimental planning for HCX", to be published in Laser and Particle Beam 20 (4), 2002.

[3] H. Qin, E. A. Startsev and R. C. Davidson, "Nonlinear Perturbative Particle Simulation Studies of the ElectronProton Two-Stream Instability in High-Intensity Proton Beams", Phys. Rev. Special Topics on Accelerators and Beams 6, 014401 (2003).

[4] T.-S. Wang, P. J. Channell, R. J. Macek and R. C. Davidson, "Centroid Theory of Transverse Electron-Proton Two-Stream Instability in a Long Proton Bunch" Phys. Rev. Special Topics Accelerators and Beams 6, 014204 (03).

[5] J. L. Vay, et. al.. "Mesh Refinement for Particle-In-Cell Plasma Simulations: applications to and benefits for Heavy Ion Fusion, to be published in Proc. of $14^{\text {th }}$ Int. Symp. Heavy Ion Inertial Fusion. (Laser and Particle Beams). [6] Frontiers in High Energy Density Physic -The X-Games of Contemporary Science (Nat Academies Press, 2003). 